\title{
Duration of Long-Term Care: Socio-Economic Factors, Type of Care Interactions and Evolution*
}

\author{
Michel Fuino, Joël Wagner ${ }^{\dagger}$
}

\begin{abstract}
The time spent in dependence and the type of care an elderly receives are the two main cost drivers of long-term care (LTC). We aim to provide a better understanding of the duration of care by using a comprehensive social insurance dataset covering the LTC needs in Switzerland over a 20-years-period and including 230000 observations on dependent elderly. First, using the framework of survival analysis, we calculate Kaplan-Meier estimates for the care duration and derive the main explaining factors through econometric models when care is received at home and in an institution. Retaining only significant covariates, the final accelerated failure time models allow us to predict the duration for different profiles of elderly along their age, gender, region of residence, type of household composition, acuity level and pre-retirement income. Second, we study the interaction of care durations when care is provided at home and in an institution. While our data supports that for short at-home care durations the time spent in institutional care is reduced, we find that both types of care are non-substitutes when the time spent at home has been longer. Under the latter regime, the time spent in institutional care remains at a constant level. Finally, given the longevity improvements over the period of observation, we analyze the impact of living longer on the time spent in dependence. Our results show that while the mean age at entry in dependence grows, the overall care duration does not significantly change. Given the expected increasing number of elderly in most developed countries, our study is relevant for government planning, budgeting social insurance schemes, estimating personal savings needs and calculating private insurance premiums.
\end{abstract}

Key words survival analysis $\cdot$ at-home care $\cdot$ institutional care $\cdot$ empirical study

${ }^{*}$ The present manuscript as of 30 August 2019 has been accepted for publication in the journal Insurance: Mathematics and Economics on 17 November 2019. Published journal article DOI: 10.1016/j.insmatheco.2019.11.004. (C)2019. This manuscript version is made available under the CC-BY-NC-ND 4.0 license.

${ }^{\dagger}$ Michel Fuino (michel.fuino@unil.ch) and Joël Wagner (joel.wagner@unil.ch) are with the Department of Actuarial Science, Faculty of Business and Economics, University of Lausanne. The second author is also with the Swiss Finance Institute, University of Lausanne, Switzerland. Financial support from the Swiss National Science Foundation (grant no. 100018_169662) and the Swiss Insurance Association is gratefully acknowledged. Support from the Swiss Central Compensation Office for providing the data is gratefully acknowledged. The authors are thankful for the comments on earlier versions of this manuscript from participants in the following conferences and seminars: Lyon-Lausanne seminar (Lausanne, Jun. 2018), European Actuarial Journal Conference (Leuven, Sept. 2018), Longevity 14 (Amsterdam, Sept. 2018), Institute of Actuaries of France Conference (Paris, May 2019), Swiss Insurance Association Conference (Lausanne, June 2019), International Congress on Insurance: Mathematics and Economics (Munich, July 2019) and American Risk and Insurance Association Annual Meeting (San Francisco, Aug. 2019). 


\section{Introduction}

The need of help in activities of daily living (ADL) for the old-age generation has surged in most developed countries (Fujisawa and Colombo, 2009). The systemic threat linked to the financing of long-term care (LTC) relies both on life span improvements and the retirement of the babyboom generation (United Nations, 2016). Longevity implies that the number of elderly reaching higher ages increases. In fact, the number of individuals aged over 80 years is predicted to be three times higher in 30 years from now (Llena-Nozal et al., 2011) and LTC prevalence rates strongly expand at ages above 80 years. Their number is predicted to increase by a factor of four in the U.S. (Brown and Finkelstein, 2009), by more than two in the U.K. (United Nations, 2015) and by 2.5 in Switzerland (Fuino and Wagner, 2018b). This development will put the current financial planning under pressure and, as things stand, no country is prepared to cope with the upcoming costs generated by these future needs. Furthermore, the existing infrastructure will not be sufficient and providing proper care will experience a lack of qualified staff (Nichols et al., 2010; World Health Organization, 2014). Indeed, the latter arguments require a review of the societal and political scope on how LTC will be handled in the future. This includes among others the question about the usage of different care options. In the U.S., a broad offer allows elderly to choose between home care, community services, assisted living, continuing care retirement communities and nursing homes (Feder et al., 2000). In France, the social system in place essentially distinguishes home-based and institutional care (Doty et al., 2015). While the distinct types of care address the needs at different stages, they leave room for individual preferences along available financial resources (Mcauley and Blieszner, 1985; Eckert et al., 2004). Currently, most countries propose very different schemes to provide and to cover the financing of LTC. For example, while in the U.S. Medicaid provides means-tested allowances financed by taxes (Brown and Finkelstein, 2008), the compulsory social scheme in Germany is based on employee and employer contributions giving access to care benefits (Schulz, 2010). The systems in place are mostly based on macro-level financial planning and budgeting social insurance schemes. However, reforming such systems and taking political decisions is difficult since very little knowledge exists on the individual level. In fact, the time spent in and the usage of LTC is heterogeneous, dependent on personal health states and driven by socio-demographic characteristics. Moreover, preferences and family situations influence the paths, i.e. the types of care requested and the length of care stays. For example, dependent elderly living close to their family may rather stay at home, while others can only rely on institutional care (Pinquart and Sörensen, 2011; Mommaerts, 2015; Choi and Joung, 2016). Uncertainty about the personal situation and available care options at higher ages makes it hard to estimate savings needs. On the one hand, this insecurity can be a trigger for LTC insurance demand (Brown and Finkelstein, 2007). On the other hand, it is challenging for insurance companies to price such insurance. In the past, such products have regularly undergone premium reassessments on the U.S. market (Nordman, 2016).

Assessing the financing needs and developing sustainable policies to address the societal issues requires detailed knowledge on the LTC duration. While most studies analyze the time spent in dependence using survey data, the contribution of our work is to provide an assessment of the LTC duration using a comprehensive longitudinal dataset. Given the available data, we apply the framework of survival analysis to better understand the time an elderly spends in LTC. Our research is guided by three objectives and we aim to

(1) identify the socio-economic factors influencing the LTC duration along types of care, 
(2) measure how the interaction of at-home and institutional care influences the overall care duration and,

(3) assess the evolution of the age at entry in and the duration of old-age dependence over the past years.

At the end, we aggregate our main findings and discuss applications for both policymakers and insurers.

We conduct our investigation using data recording the total old-age dependent population in Switzerland. The data covers the period from 1995 to 2015 and provides about 230000 observations on dependent elderly (see also, Fuino and Wagner, 2018a,b). Each record includes information on the type of care and on the dates when care is received. Further variables inform about the age, the gender, the canton, the civil status, the acuity level, the pre-retirement income and the nationality. Given the longitudinal data structure, we account for right-censoring and use Kaplan-Meier estimates (Kaplan and Meier, 1958) for the descriptive statistics. To understand the duration in detail, we build on survival analysis to derive an econometric model. Thereby, the "survival" time is linked to the time spent in a given type of LTC. Those durations are best fitted with a Gamma distribution. We develop accelerated failure time (AFT, Wei, 1992) models for identifying the variables that correlate with the time spent in dependence. Such models have been used in a similar context by Lee et al. (2017) when investigating the risk factors affecting Alzheimer's disease. Since costs highly diverge along types of care (Czado and Rudolph, 2002, Costa-Font and Courbage, 2012), we specify one model for the overall care duration and two separate models for the time spent in at-home respectively institutional care. By estimating the effect of individual socio-demographic factors, we add on previous studies that have identified the age and the gender (e.g., Mathers, 1996, Germain et al., 2016), the region of residence (Gentili et al., 2017), the type of household (Freedman and Martin, 1998), the acuity level and the pre-retirement income (Fried et al., 2001; Mobley et al., 2006; Planchet et al., 2018) as relevant determinants of the duration. To make our models applicable for predicting LTC durations, we use a stepwise forward selection of the variables and only retain significant covariates (Venables and Ripley, 2002). Thereafter, we analyze the interaction between care received at home and in an institution as well as their effect on the overall duration. We assess if care provided at home can substitute institutional care (Kaye et al., 2009; Helmchen and Lo Sasso, 2015) or act as a complement. The developed duration models allow to detail the study of the interaction effects between types of care. We measure substitution effects and determine to what extent the care duration is incompressible. Finally, we assess potential changes in the age at entry and the period length spent in dependence. While longevity improvements are a great achievement for the society, its impact on when elderly become dependent and on the time spent in dependence is only shortly studied. Currently, two opposite theories exist (Pellegrini et al., 2006; Payne et al., 2007). While Fries $(1980,1989,2005)$ finds that gains of life expectancy result in a reduction of the time spent in dependence, works by Kramer (1980), Olshansky et al. (1990) and Gruenberg (2005) state that the time spent in dependence will rather expand.

Our main results are threefold. First, we find that all variables, except nationality, are significant factors determining the time spent in LTC. The age when entering dependency, the gender and the acuity level are the most important in our models. We predict that eighty years old men remain on average about three years and a half in dependence while at the age of ninety years they only stay two years and a half which is linked to higher mortality. Conversely, we 
observe that women experience on average one year more in dependence when compared to men. Higher acuity levels shorten the LTC duration. Further, elderly living in the Germanspeaking part of Switzerland spend less time in dependence than elderly living in the Frenchand Italian-speaking regions. Such result is related to both local policy and cultural aspects. Our results show that the pre-retirement income, i.e. a proxy of the social status, only has a small while still significant effect on the duration of LTC. Second, focusing on the durations by types of care, we discover the existence of both a substitution and a non-substitution effect between both types. At the beginning of dependence, we note a substitution effect with home care reception strongly reducing the length of stays in institutional care. Afterwards, longer home care duration no more reduces the length of institutional care. This non-substitution effect highlights the non-reducible part of care in an institution. This result addresses an important concern for governance since it shows that maintaining dependent elderly at home can be both beneficial in terms of reducing costs and fulfilling the preference of many elderlies. Third, we show that over the observation period the mean age at entry in dependence shifts by about one year towards higher ages, from 81 to 82 years for men and from 85 to 86 years for women. Further, we observe that the overall time spent in dependence does not significantly change, wherefore, we argue that the gain of life expectancy does not induce longer stays in dependence.

This article is organized as follows: In Section 2, we describe the LTC system in Switzerland, introduce the available data and present descriptive statistics on the overall, the at-home and the institutional LTC duration. In Section 3, we derive the econometric models that lay the basis for the duration analysis. Then, we present our results along the three research objectives in Section 4. In Section 5, we predict the LTC duration for selected profiles and discuss perspectives for the insurance industry. We conclude in Section 6.

\section{Longitudinal data on LTC in Switzerland}

In order to address the research objectives laid out in the Introduction, we rely on comprehensive data covering the old-age population in need of care in Switzerland. In the following, we describe the Swiss LTC system (Section 2.1) and we lay out the available data (Section 2.2) that allows us to study the time spent in dependence. In Section 2.3, we discuss the right-censoring present in our data and provide Kaplan-Meier estimates for the duration. Finally, in Section 2.4, we report descriptive statistics on the median duration.

\subsection{LTC system in Switzerland}

Switzerland is a federal state divided in 26 cantons and three linguistic regions. About two thirds of the population lives in the German-speaking region, one quarter in the French-speaking region and the remainder in the Italian-speaking region. Inhabitants of this highly developed country present a life expectancy of 83.0 years well above the OECD average of 80.6 years of life expectancy at birth (OECD, 2017). The demand for old-age LTC, i.e. care provided to elderly over 65 years by institutions and professionals is predicted to increase by a factor of 2.45 in the period from 2015 to 2045 (Fuino and Wagner, 2018b). When considering the overall costs from formal care at home and in institutions, about ten percent of the LTC expenditures are covered by the state, thirty percent are taken over by mandatory social health insurance and the remaining sixty percent are left to the households (Swiss Re, 2014). The government provides non-means tested allowances for elderly in need of assistance with ADL regulated under the 
first pillar of the Old-Age Social Insurance (OASI) law. Three acuity levels are distinguished and higher amounts are paid for more severe dependence in line with existing limitations in ADL. In the Swiss social system, the considered ADL are (1) dressing and undressing, (2) getting up, sitting and lying down, (3) eating, (4) personal hygiene, (5) using the toilet and (6) mobility. Mild acuity identifies persons in need of regular assistance with at least two ADL or permanent personal supervision. Moderate acuity characterizes dependents needing assistance in at least two ADL and permanent personal supervision. Persons in need of regular assistance in all the ADLs are classified severely dependent (Becker and Reinhard, 2018). Under OASI law, the mandatory health insurance reimburses medication to avoid health deterioration and pays for selected medical equipment as well as care at home. Finally, the households pay for the remaining costs which in the case of institutional care essentially comprise the accommodation related services like lodging and meals.

\subsection{Description of the available dataset}

Our study is based on a longitudinal social insurance dataset covering LTC needs in Switzerland as recorded by the OASI allowances paid to dependent elderly. The data provides a comprehensive and representative view of the total LTC demand since any elderly aged $65+$ years living in Switzerland and recognized as mildly, moderately or severely dependent is eligible for receiving the allowance. The medical assessment is done through the completion of a two-page form under the control of a recognized entity. The state at-home care provider evaluates the level of dependence of elderly being cared for at home, and institutions manage the request for persons receiving inpatient care. There is no specific routine defining a reassessment date, i.e. when significant changes in the dependence level are observed by the above bodies, a reassessment is made. While the same process applies throughout Switzerland, the amount of the allowance varies among cantons. Records from the OASI allowance quasi cover the total dependent population in Switzerland. Nevertheless, since dependencies are recorded only when an allowance is paid, i.e. after a request is made, there are limitations to full coverage. For example, elderly persons living at home may sometimes be unaware of the benefits they are entitled to or may forget to request them despite being eligible.

The original data $\mathcal{D}_{1}$ compiled by the Swiss Central Compensation Office $(\mathrm{CCO})^{1}$ contains information for the period from 1995 to 2015 on the age, the gender, the canton of residence, the civil status, the acuity level and the type of care elderly have received (see also Fuino and Wagner, 2018a). While the acuity levels include the three classes mild, moderate and severe as introduced above, the types of care distinguished are at-home care (HC) and institutional care (IC). In the longitudinal data, the start and end dates for each acuity level and type of care as well as death are reported at a precision of one month (Fuino and Wagner, 2018b). For calculating the overall duration in dependence $D$, we evaluate the number of months between the respective start and end dates, i.e. dates of entry in dependence and of death. In the same way, the durations $D^{\mathrm{HC}}$ and $D^{\mathrm{IC}}$ denote the time spent in care provided at home and in an institution, respectively. From the data we derive the age where the elderly becomes dependent $(A G)$ and the gender $(G E)$. While we find age entries between 65 and 108 years, we restrict our observations to ages starting at 66 years for both men and women, removing dependent persons that were disabled before the statutory retirement age (cf. Fuino and Wagner, 2018a, Sect. 3.1). Combining the age information with the types of care, we specifically derive the age where the

\footnotetext{
${ }^{1}$ ww. zas.admin. ch
} 
elderly receives at-home care $\left(A G^{\mathrm{HC}}\right)$ or institutional care $\left(A G^{\mathrm{IC}}\right)$ for the first time. Using the reported cantons of residence, we build three linguistic region clusters along the German-, French- and Italian-speaking parts of Switzerland $(L R) .^{2}$ While the civil status is reported in terms of nine categories, ${ }^{3}$ we define a type of household variable $(H H)$ by consolidating them into two groups: the two-persons households containing the "married" and the "registered partner" categories and the single-person households incorporating all other categories. From the recorded acuity levels, we derive the first acuity level $(A L)$, i.e. the level registered when the individual starts receiving OASI allowances. Finally, we construct a categorical variable along the types of care $(T C)$ identifying the paths of elderly along three alternatives: reception of home-based care only, of institutional care only and of both at-home and institutional care. ${ }^{4}$ We summarize the introduced variables in Table 1.

The final data $\mathcal{D}_{1}$ used in our analysis accounts for 229117 elderly becoming dependent. More specifically, we observe 20069 persons entering at-home care and 216520 receiving institutional care. Therefrom, 7472 elderly receive both at-home and institutional care. In most of the records we are able to observe the complete dependence period from entry in dependence till death and thus to calculate the overall LTC duration D. However, given our data is longitudinal, part of the available observations (19.8\% in the case of the overall duration) are incomplete and subject to right-censoring (see Section 2.3 for a detailed discussion and statistics). The number of available observations exceeds the data used in other LTC studies like, for example, Biessy (2016) where a total of 20988 records from a French private insurer are studied. Furthermore, recall that our social insurance dataset gives a representative picture of the total dependent population in Switzerland, while most other current studies focus on private insurance datasets (e.g., D'Amico et al., 2009).

As an extension of the above data, the $\mathrm{CCO}$ is able to provide information on the last recorded income before retirement and nationality of a selected number of elderly. Information on the preretirement income is related to the salary on which compulsory first pillar OASI contributions are paid (SFIO, 2018) and is only available for elderly who retired before 1981. In order to include the socio-economic status in our study, we construct a measure of the salary received before retirement $(S A)$ based on the individuals' available income history. ${ }^{5}$ In fact, the preretirement income can serve as a proxy of the wealth and the social class an individual belongs to. To account for inflation and structural income changes, we report each income information to their value in 2015 by using the Swiss consumer price index. ${ }^{6}$ Further, the additional data

\footnotetext{
${ }^{2}$ Three large linguistic regions are distinguished in Switzerland. These regions are (1) the German-speaking region comprising the cantons of Aargau, Appenzell Innerrhoden, Appenzell Ausserrhoden, Bern, Basel-Landschaft, Basel-Stadt, Glarus, Graubünden, Luzern, Nidwalden, Obwalden, St. Gallen, Schaffhausen, Solothurn, Schwyz, Thurgau, Uri, Zug, and Zürich; (2) the French-speaking region comprising the cantons of Fribourg, Genève, Jura, Neuchâtel, Vaud, and Valais; and (3) the Italian-speaking region formed by the canton of Ticino.

${ }^{3}$ The reported civil status categories in the CCO data are single, married, widower, divorced, separated by judicial decision, registered partner, dissolved partnership between persons of the same sex, dissolved partnership due to the death of one partner and separated by judicial decision for persons of the same sex.

${ }^{4}$ While our study focuses on at-home and institutional care, we do not consider recovery in terms of decreasing acuity levels since our data reports very few such records. In fact, we leave out records where at-home care follows institutional care or where recovery to lower acuity levels is observed. These records make up less than $0.5 \%$ of the data (see also Fuino and Wagner, 2018a,b).

${ }^{5}$ For individuals with more than two years of information on pre-retirement income, we consider the mean between the 25\%- and 75\%-quantile of the income history. For elderly with exactly two records, we take the mean of both income figures. For the ones having only one record available, we use the figure from that single year.

${ }^{6}$ https://www.bfs.admin.ch
} 


\begin{tabular}{llcc}
\hline Variable & Description & $\mathcal{D}_{1}$ & $\mathcal{D}_{2}$ \\
\hline$D$ & Overall duration in dependence (in months) & $\checkmark$ & $\checkmark$ \\
$D^{\mathrm{HC}}$ & Duration of care at home (in months) & $\checkmark$ & $\checkmark$ \\
$D^{\mathrm{IC}}$ & Duration of care in an institution (in months) & $\checkmark$ & $\checkmark$ \\
\hline$A G$ & Age at entry in dependence: from 66 to 108 (integer values) & $\checkmark$ & $\checkmark$ \\
$A G^{\mathrm{HC}}$ & Age where care at home is received for the first time & $\checkmark$ & $\checkmark$ \\
$A G^{\mathrm{IC}}$ & Age where care in an institution is received for the first time & $\checkmark$ & $\checkmark$ \\
$G E$ & Gender: male, female & $\checkmark$ & $\checkmark$ \\
$L R$ & Linguistic region: German, French, Italian & $\checkmark$ & $\checkmark$ \\
$H H$ & Household composition: single person, two persons & $\checkmark$ & $\checkmark$ \\
$A L$ & Acuity level at entry: mild, moderate, severe & $\checkmark$ & $\checkmark$ \\
$T C$ & Types of care received: HC only, IC only, combination of HC and IC & $\checkmark$ & $\checkmark$ \\
$S A$ & Pre-retirement income (in CHF) & & $\checkmark$ \\
$N A$ & Nationality: Swiss, Austrian, French, German, Italian and Other & & $\checkmark$ \\
\hline
\end{tabular}

Note: "HC" stands for at-home care, "IC" stands for institutional care.

Table 1: Description of the variables included in the datasets $\mathcal{D}_{1}$ and $\mathcal{D}_{2}$.

reports information on the last recorded nationality of the elderly. We observe more than twenty nationalities that we cluster into six categories $(N A)$. Thereby, we build five categories along Switzerland and its neighboring countries considering Swiss, Austrian, French, German and Italian citizens separately. A sixth category includes all other nationalities. In our study, we aim to include both covariates that add information on 92898 of the observations recorded in $\mathcal{D}_{1}$. We construct an augmented dataset $\mathcal{D}_{2}$, a subset of the observed elderly included in $\mathcal{D}_{1}$, by merging the additional information with $\mathcal{D}_{1}$ and retaining only the complete records. Table 1 reports the variables that are included in both datasets.

\subsection{Right-censored data and Kaplan-Meier estimates}

Right-censoring With the coverage of the longitudinal data $\mathcal{D}_{1}$ and $\mathcal{D}_{2}$ stopping in 2015, many dependents are still alive and in dependence on the last record date. This makes a significant amount of records affected by non-informative right-censoring, i.e. by censoring that is not the consequence of a particular event. In Table 2, we report the number of observations $N$ that are available for assessing the overall care duration $D$, the duration of care at home $D^{\mathrm{HC}}$ and the duration of care in an institution $D^{\mathrm{IC}}$. We specify how many records and what percentage are affected by censoring in both datasets $\mathcal{D}_{1}$ and $\mathcal{D}_{2}$. In the data $\mathcal{D}_{1}, 19.8 \%$ of the observations for the overall duration are affected by right-censoring. They are $48.2 \%$ for the at-home care duration and $16.5 \%$ for the institutional care duration. In the data $\mathcal{D}_{2}$, we find that $32.4 \%$, $50.1 \%$ and $27.5 \%$ of the records are censored.

Further, in Table 3, we detail the shares of uncensored and censored records and lay out the total number of observations $N$ for the overall care duration $D$ in the data $\mathcal{D}_{1}$ by calendar year. Thereby, the calendar year refers to the year when the recorded individual becomes dependent. Most of the years and especially those at the beginning of the observation period expectedly show low levels of right-censored data. However, for the years after 2010 the percentage of rightcensoring becomes very important exceeding $50 \%$ in 2013 . On the one hand, this can be related to the relatively short remaining period of observation for persons entering in dependence af- 


\begin{tabular}{|c|c|c|c|c|c|c|c|c|c|c|c|c|}
\hline & \multicolumn{4}{|c|}{$D$} & \multicolumn{4}{|c|}{$D^{\mathrm{HC}}$} & \multicolumn{4}{|c|}{$D^{\mathrm{IC}}$} \\
\hline & \multicolumn{2}{|c|}{$\mathcal{D}_{1}$} & \multicolumn{2}{|c|}{$\mathcal{D}_{2}$} & \multicolumn{2}{|c|}{$\mathcal{D}_{1}$} & \multicolumn{2}{|c|}{$\mathcal{D}_{2}$} & \multicolumn{2}{|c|}{$\mathcal{D}_{1}$} & \multicolumn{2}{|c|}{$\mathcal{D}_{2}$} \\
\hline & $N$ & $(\%)$ & $N$ & $(\%)$ & $N$ & $(\%)$ & $N$ & $(\%)$ & $N$ & $(\%)$ & $N$ & $(\%)$ \\
\hline Uncensored & 183752 & $\overline{(80.2)}$ & 62840 & $\overline{(67.6)}$ & $\overline{10401}$ & $\overline{(51.8)}$ & 6923 & $\overline{(49.9)}$ & 180820 & $\overline{(83.5)}$ & 60900 & $(72.5)$ \\
\hline Censored & 45365 & $(19.8)$ & 30058 & $(32.4)$ & 9668 & $(48.2)$ & 6948 & $(50.1)$ & 35700 & $(16.5)$ & 23111 & $(27.5)$ \\
\hline Total & 229117 & $\overline{(100)}$ & 92898 & $\overline{(100)}$ & 20069 & $\overline{(100)}$ & 13871 & $\overline{(100)}$ & 216520 & $\overline{(100)}$ & 84011 & $(100)$ \\
\hline
\end{tabular}

Table 2: Number $N$ and shares (in \%) of uncensored and censored records for the overall $D$, at-home $D^{\mathrm{HC}}$ and institutional $D^{\mathrm{IC}}$ care durations in the data $\mathcal{D}_{1}$ and $\mathcal{D}_{2}$.

ter 2010 (less than five years). On the other hand, since 2011, the Federal Office of Public Health allows benefits for mildly dependent persons that are cared at home (FOPH, 2013) implying an increase in records with individuals at the mild acuity level and in at-home care. The latter may, under certain circumstances, exhibit longer care durations (Dutoit et al., 2016).

\begin{tabular}{lrrrrrrrrrrr}
\hline & 1995 & 1996 & 1997 & 1998 & 1999 & 2000 & 2001 & 2002 & 2003 & 2004 & 2005 \\
\hline Uncensored (\%) & 99.7 & 99.7 & 99.6 & 99.5 & 99.2 & 99.0 & 98.8 & 98.5 & 97.9 & 96.9 & 96.4 \\
Censored (\%) & 0.3 & 0.3 & 0.4 & 0.5 & 0.8 & 1.0 & 1.2 & 1.5 & 2.1 & 3.1 & 3.6 \\
\hline$N$ & 8288 & 8521 & 9197 & 9313 & 9523 & 10364 & 10711 & 10506 & 10809 & 11074 & 11008 \\
& & & & & & & & & & & \\
& 2006 & 2007 & 2008 & 2009 & 2010 & 2011 & 2012 & 2013 & 2014 & 2015 & Total \\
\hline Uncensored (\%) & 95.1 & 92.9 & 89.6 & 85.5 & 80.0 & 60.9 & 53.1 & 38.6 & 21.3 & 9.8 & 80.2 \\
Censored (\%) & 4.9 & 7.1 & 10.4 & 14.5 & 20.0 & 39.1 & 46.9 & 61.4 & 78.7 & 90.2 & 19.8 \\
\hline$N$ & 10719 & 10494 & 10574 & 11148 & 11719 & 19631 & 13467 & 13759 & 12955 & 5337 & 229117 \\
\hline
\end{tabular}

Table 3: Number $N$ and shares (in \%) of uncensored and censored records for the overall care duration $D$ in the data $\mathcal{D}_{1}$ by calendar year of entry in dependence.

Figure 1 focuses on the durations that are censored. The graphs lay out the probability distribution of the different censored durations $D, D^{\mathrm{HC}}$ and $D^{\mathrm{IC}}$ from both datasets $\mathcal{D}_{1}$ and $\mathcal{D}_{2}$. We observe that the distributions from both datasets $\mathcal{D}_{1}$ and $\mathcal{D}_{2}$ are similar. We note two patterns that emerge from the graphs. First, in all distributions, we observe more records at yearly durations, i.e. at $12,24,36,48$ and 60 months. This can be partly explained by the health reassessment that is often done yearly in practice. Second, we note that the duration in at-home care $D^{\mathrm{HC}}$ presents a high proportion of censored data at 60 months with only few observations beyond that threshold. In fact, while care at home for moderately and severely dependent elderly is recognized since 2001, benefits for mildly dependent persons that are cared at home are paid only since 2011 (see above). Further, since at-home care is mostly provided to mildly dependent persons, the observed spike at 60 months can directly be linked to the observation period of five years between the last year of records (2015) and the year when at-home care for mildly dependent has been recognized (2011). The few observations with durations beyond 60 months stem from moderately and severely dependent persons.

Kaplan-Meier estimates Given the right-censoring of the records in our data, when performing descriptive statistics on the duration, calculating mean values is no longer adequate and using the median is the method of choice. A standard way to calculate the median is to apply Kaplan-Meier estimation on the observed durations. The estimator has been developed by (Kaplan and Meier, 1958) and details about its evaluation and the calculation of confidence 

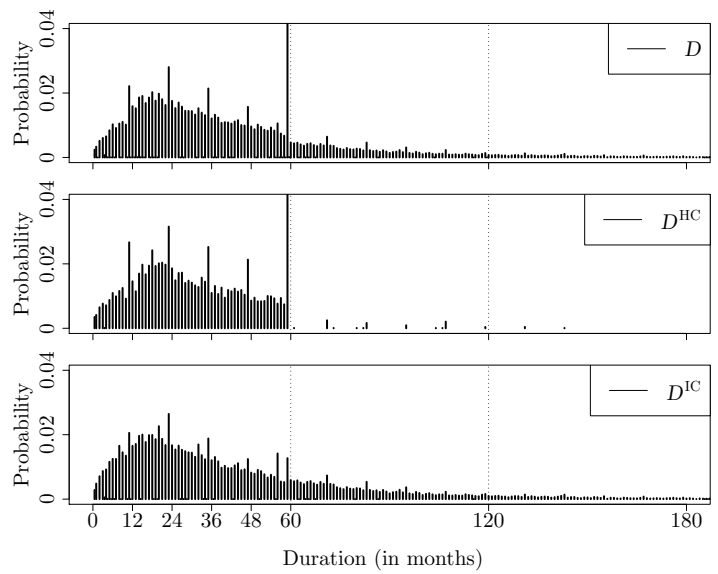

(a) Data $\mathcal{D}_{1}$
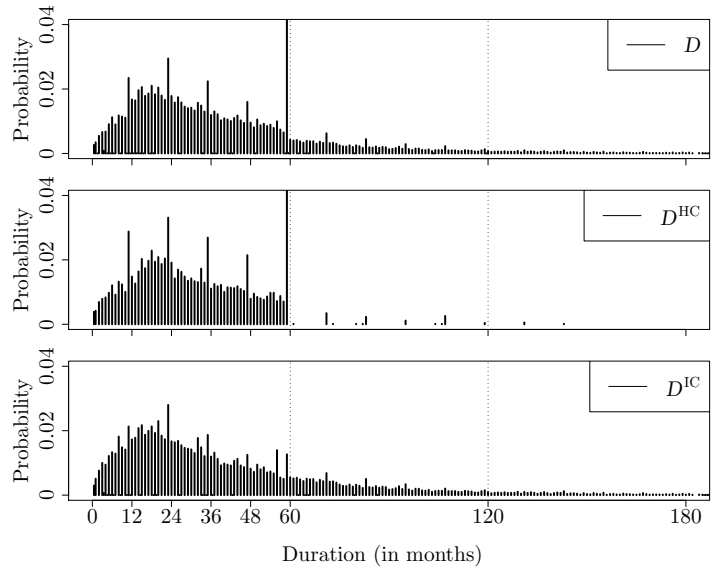

(b) Data $\mathcal{D}_{2}$

Figure 1: Probability distributions of the censored overall $D$, at-home $D^{\mathrm{HC}}$ and institutional $D^{\mathrm{IC}}$ care durations in the data $\mathcal{D}_{1}$ and $\mathcal{D}_{2}$.

intervals can be found, for example, in Aalen et al. (2008). The Kaplan-Meier estimate provides a non-parametric computation of the survival curve. Thereby, we employ the term "survival" time for identifying the time spent in a state where one type of care is provided before leaving it for another type of care or death. The curve from the Kaplan-Meier estimate describes the probability distribution of the duration $D, D^{\mathrm{HC}}$ or $D^{\mathrm{IC}}$ a dependent elderly receives LTC. From that distribution we compute the median duration in dependence, i.e. the duration after which 50 percent of the individuals have changed type of care or died. In the case where rightcensored records dominate our data, the median will not exist in all cases.

Using the data $\mathcal{D}_{1}$, we present in Figure 2 the Kaplan-Meier estimates for (a) the overall care duration $D$, (b) the at-home care duration $D^{\mathrm{HC}}$ and (c) the institutional care duration $D^{\mathrm{IC}}$. In each graph we compare the estimate obtained from the complete set of records in $\mathcal{D}_{1}$ (plain curve, "all records") to the estimate obtained when using the subset of uncensored observations only (dashed curve, "uncensored records"). As expected, we observe that the estimate including the right-censored records is shifted towards higher durations when compared to the estimate from uncensored records. We observe very similar shapes for the overall and for the institutional care durations. For the at-home care duration, the 95\% confidence interval (gray shaded area) becomes wider after a duration of about 60 months. This blur comes from the important rightcensoring observed for stays at home and the small number of observations in that area (cf. Table 2). The important share of right-censored data in the case of the at-home care duration also explains the larger difference between the estimates on the data including respectively excluding censored records. Considering the $50 \%$ survival probability (horizontal dashed line on the graphs), we find that the median overall duration $m_{D}$ is 33 months (considering all records). The median duration for at-home care $m_{D^{\mathrm{HC}}}$ is 34 months and for institutional care we have $m_{D^{\mathrm{IC}}}=32$ months.

\subsection{Statistics on the median care duration}

To provide descriptive statistics on the LTC duration observed in our data, we apply KaplanMeier estimates on subsets of the data along the values of the covariates of interest. From the estimates we then derive the median durations per category. In Table 4, we present the me- 


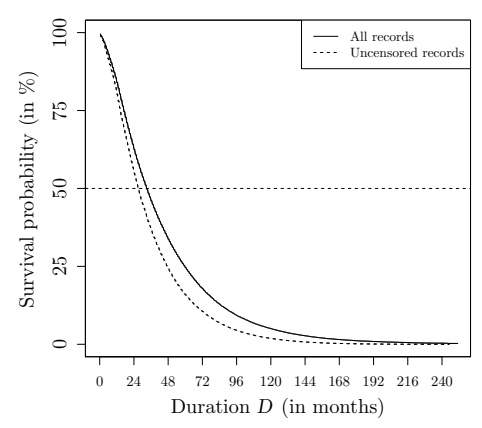

(a) $D$

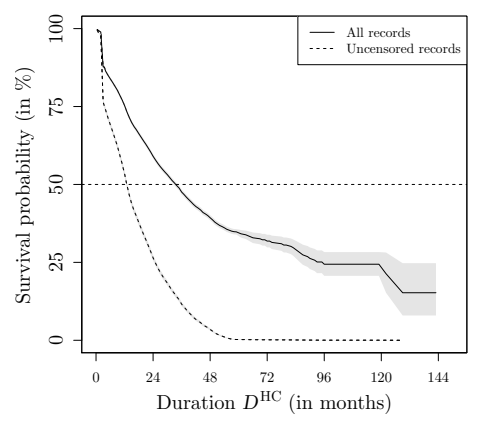

(b) $D^{\mathrm{HC}}$

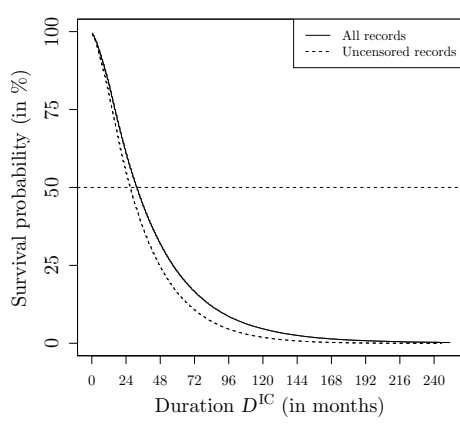

(c) $D^{\text {IC }}$

Note: From the intersection between the survival curve and the horizontal dashed line at a survival probability value of $50 \%$ the median time can be read. The gray shaded area indicates the $95 \%$ confidence interval of the estimate.

Figure 2: Kaplan-Meier estimates of the survival curve for the overall $D$, at-home $D^{\mathrm{HC}}$ and institutional $D^{\mathrm{IC}}$ care durations in the data $\mathcal{D}_{1}$.

dian times of overall $\left(m_{D}\right)$, at-home $\left(m_{D^{\mathrm{HC}}}\right)$ and institutional care $\left(m_{D^{\mathrm{IC}}}\right)$ along the variables available in both data $\mathcal{D}_{1}$ and $\mathcal{D}_{2}$ (cf. Table 1 ). We consider the age at entry, the gender, the linguistic region, the type of household, the acuity level at entry, the reception of at home or institutional care, the pre-retirement income and the nationality. For the continuous variables age and income, we introduce categories to report the statistics. For the age at entry in dependence, we consider five categories by decades regrouping the ages 66 - 69, $70-79,80-89$, 90 - 99 years and above 100 years. For the pre-retirement income, we build four categories using the quartiles (CHF 22308,49538 and 77134 ) of the income distribution when considering the overall care duration $D$ in the data $\mathcal{D}_{2}$. We keep the same categories when describing the durations $D^{\mathrm{HC}}$ and $D^{\mathrm{IC}}$. In our statistics, we report the median duration and the share of observations in parentheses for each class.

Let us first comment on the overall care duration $D$ recorded in the data $\mathcal{D}_{1}$. We observe that the time spent in dependence decreases with the age classes. For example, the median duration $m_{D}$ is 66 months for ages between 66 and 69 years and reduces to 25 months in the group of the nonagenarians. The age class of the octogenarians contains the largest number of observations with about half of the records. We note an important difference in the median duration and share when comparing both genders. Women represent about two thirds of the records in the data $\mathcal{D}_{1}$ and display a median overall time in dependence $m_{D}$ of 35 months. This figure is only 29 months in men. The distribution of the dependent elderly among linguistic regions follows the one of the total population published by the Swiss Federal Statistical Office (FSO). ${ }^{7}$ In fact, they are about two thirds (66.8\%) living in the German-speaking region, $25.9 \%$ in the French- and $7.3 \%$ in the Italian-speaking regions. Dependent residents from the Italian-speaking region show a high median duration $m_{D}$ of 44 months while in the German- and the Frenchspeaking regions we observe durations at lower levels of 32 and 35 months, respectively. For the type of household, we identify most of the records in single person households (68.0\%). While this applies for the duration in both overall and institutional care, the share reduces to $55.7 \%$ for the at-home care duration data. Finally, comparing at-home and institutional care, the elderly

\footnotetext{
${ }^{7}$ www.bfs.admin.ch
} 


\begin{tabular}{|c|c|c|c|c|c|c|c|c|c|c|c|c|}
\hline & \multicolumn{4}{|c|}{$D$} & \multicolumn{4}{|c|}{$D^{\mathrm{HC}}$} & \multicolumn{4}{|c|}{$D^{\mathrm{IC}}$} \\
\hline & \multicolumn{2}{|c|}{$\mathcal{D}_{1}$} & \multicolumn{2}{|c|}{$\mathcal{D}_{2}$} & \multicolumn{2}{|c|}{$\mathcal{D}_{1}$} & \multicolumn{2}{|c|}{$\mathcal{D}_{2}$} & \multicolumn{2}{|c|}{$\mathcal{D}_{1}$} & \multicolumn{2}{|c|}{$\mathcal{D}_{2}$} \\
\hline & $m_{D}$ & $(\%)$ & $m_{D}$ & $(\%)$ & $m_{D^{\mathrm{HC}}}$ & $(\%)$ & $m_{D^{\mathrm{HC}}}$ & $(\%)$ & $m_{D^{\mathrm{IC}}}$ & $(\%)$ & $m_{D^{\mathrm{IC}}}$ & $(\%)$ \\
\hline \multicolumn{13}{|l|}{ Age at entry } \\
\hline $66-69$ & 66 & $(3.7)$ & 63 & $(8.0)$ & 78 & $(6.8)$ & 74 & $(9.3)$ & 61 & $(3.4)$ & 58 & $(7.5)$ \\
\hline $70-79$ & 46 & $(21.4)$ & 44 & $(38.5)$ & 44 & $(26.8)$ & 44 & $(33.6)$ & 44 & $(21.0)$ & 41 & $(38.7)$ \\
\hline $80-89$ & 33 & $(49.6)$ & 33 & $(47.3)$ & 33 & $(49.6)$ & 32 & $(48.4)$ & 32 & $(49.6)$ & 30 & $(47.4)$ \\
\hline $90-99$ & 25 & $(24.6)$ & 26 & $(6.2)$ & 23 & $(16.5)$ & 20 & $(8.7)$ & 24 & $(25.3)$ & 23 & $(6.4)$ \\
\hline $100+$ & 20 & $(0.7)$ & & - & 16 & $(0.3)$ & & - & 20 & $(0.7)$ & & - \\
\hline \multicolumn{13}{|l|}{ Gender } \\
\hline Male & 29 & $(32.5)$ & 33 & $(57.7)$ & 27 & $(35.6)$ & 27 & $(49.8)$ & 28 & $(32.4)$ & 31 & $(58.9)$ \\
\hline Female & 35 & $(67.5)$ & 47 & $(42.3)$ & 38 & $(64.4)$ & 47 & $(50.2)$ & 34 & $(67.6)$ & 42 & $(41.1)$ \\
\hline \multicolumn{13}{|c|}{ Linguistic region } \\
\hline German & 32 & $(66.8)$ & 36 & $(68.3)$ & 30 & $(68.9)$ & 31 & $(70.2)$ & 30 & $(66.8)$ & 34 & $(68.5)$ \\
\hline French & 35 & $(25.9)$ & 40 & $(25.1)$ & 45 & $(26.7)$ & 49 & $(25.6)$ & 33 & $(25.7)$ & 37 & $(24.7)$ \\
\hline Italian & 44 & $(7.3)$ & 49 & $(6.6)$ & 38 & $(4.4)$ & 39 & $(4.2)$ & 42 & $(7.5)$ & 47 & $(6.8)$ \\
\hline \multicolumn{13}{|c|}{ Type of household } \\
\hline Single person & 33 & $(68.0)$ & 39 & $(50.3)$ & 37 & $(55.7)$ & 42 & $(49.1)$ & 31 & $(68.6)$ & 36 & $(50.0)$ \\
\hline Two persons & 35 & $(32.0)$ & 37 & $(49.7)$ & 30 & $(44.3)$ & 29 & $(50.9)$ & 33 & $(31.4)$ & 34 & $(50.0)$ \\
\hline \multicolumn{13}{|c|}{ Acuity level at entry } \\
\hline Mild & 77 & $(8.8)$ & 86 & $(14.9)$ & 34 & $(99.1)$ & 34 & $(98.9)$ & 32 & $(3.5)$ & 33 & $(6.1)$ \\
\hline Moderate & & $(50.3)$ & 39 & $(51.7)$ & 85 & $(0.6)$ & 85 & $(0.7)$ & 36 & $(53.2)$ & 39 & $(57.0)$ \\
\hline Severe & 28 & $(40.9)$ & 31 & $(33.4)$ & 64 & $(0.3)$ & 64 & $(0.4)$ & 28 & $(43.3)$ & 31 & $(36.9)$ \\
\hline \multicolumn{13}{|c|}{ Received at-home care } \\
\hline No & 32 & $(91.2)$ & 35 & $(85.1)$ & & - & & - & 32 & $(96.5)$ & 35 & $(94.1)$ \\
\hline Yes & 74 & $(8.8)$ & 83 & $(14.9)$ & 36 & $(100)$ & 36 & $(100)$ & 31 & $(3.5)$ & 31 & $(5.9)$ \\
\hline \multicolumn{13}{|c|}{ Received institutional care } \\
\hline No & n.a. & $(5.5)$ & n.a. & $(9.6)$ & n.a. & $(62.8)$ & n.a. & $(64.1)$ & & - & & - \\
\hline Yes & 32 & $(94.5)$ & 36 & $(90.4)$ & 11 & $(37.2)$ & 10 & $(35.9)$ & 32 & $(100)$ & 35 & $(100)$ \\
\hline \multicolumn{13}{|c|}{ Pre-retirement income } \\
\hline Below 22308 & & & 34 & $(25.0)$ & & & 28 & $(25.2)$ & & & 31 & $(25.2)$ \\
\hline $22308-49538$ & & & 45 & $(25.0)$ & & & 42 & $(26.1)$ & & & 41 & $(24.8)$ \\
\hline $49539-77134$ & & & 40 & $(25.0)$ & & & 41 & $(25.2)$ & & & 37 & $(24.9)$ \\
\hline Over 77134 & & & 35 & $(25.0)$ & & & 34 & $(23.5)$ & & & 32 & $(25.1)$ \\
\hline \multicolumn{13}{|l|}{ Nationality } \\
\hline Swiss & & & 37 & $(86.7)$ & & & 34 & $(84.6)$ & & & 34 & $(87.0)$ \\
\hline Italian & & & 48 & $(6.2)$ & & & 41 & $(6.9)$ & & & 44 & $(6.1)$ \\
\hline German & & & 41 & $(2.2)$ & & & 36 & $(2.5)$ & & & 38 & $(2.2)$ \\
\hline Austrian & & & 45 & $(0.9)$ & & & 28 & $(1.1)$ & & & 40 & $(0.9)$ \\
\hline French & & & 43 & $(0.8)$ & & & 89 & $(1.0)$ & & & 37 & $(0.8)$ \\
\hline Other & & & 47 & $(3.2)$ & & & 50 & $(3.9)$ & & & 43 & $(3.1)$ \\
\hline Overall & 33 & $(100)$ & 38 & $(100)$ & 34 & $(100)$ & 36 & $(100)$ & 32 & $(100)$ & 35 & $(100)$ \\
\hline$N$ & & 229117 & & 92898 & & 20069 & & 13871 & & 216520 & & 84011 \\
\hline
\end{tabular}

Note: Durations are expressed in months. Relative frequencies (shares in \%) are reported in parenthesis. "_" are zero values and "n.a." stands for not applicable.

Table 4: Descriptive statistics on the median overall $D$, at-home $D^{\mathrm{HC}}$ and institutional $D^{\mathrm{IC}}$ care durations in the data $\mathcal{D}_{1}$ and $\mathcal{D}_{2}$.

receiving at-home care have entered dependence with a mild acuity level (99.1\%). In our data, most of the elderly receiving institutional care have entered dependence with moderate $(53.2 \%)$ or severe dependence $(43.3 \%)$ and have not received at-home care before $(96.5 \%)$.

When looking at the data $\mathcal{D}_{2}$, we observe an important change in the shares of observations 
by age classes and by gender. The shares of elderly that are in their nineties are much lower (compare, for example, the values of $24.6 \%$ and $6.2 \%$ ) while younger observations appear more frequently. In the data $\mathcal{D}_{2}$, the shares of dependent men have also risen to above half of the records (e.g., $57.7 \%$ against $32.5 \%$ in $\mathcal{D}_{1}$ ). Those changes are linked to the selection of the records retained in the data $\mathcal{D}_{2}$ that is based on the availability of OASI income information, more frequently available for men than for women given gender differences in the composition of the labor market. With regard to the income classes we note that the median care duration decreases with increasing pre-retirement income. Most of the observed population receiving allowances is Swiss (86.7\%), followed by the Italian (6.2\%), the German (2.2\%), the Austrian (0.9\%) and the French (0.8\%) citizens residing in Switzerland.

\section{Model framework}

Before specifying the econometric models in Section 3.2, we review various aspects relating to our modeling approach. Thereby, we study the distribution of the care duration, discuss the explanatory variables and lay out the model setup and MLE estimation in Section 3.1.

\subsection{Modeling approach}

Distribution of the care duration With the aim of setting up regression models with the LTC duration as a dependent variable, we start by studying the distribution of the variables of interest, namely the overall $D$, the at-home $D^{\mathrm{HC}}$ and the institutional $D^{\mathrm{IC}}$ care durations, as recorded in both data $\mathcal{D}_{1}$ and $\mathcal{D}_{2}$. We assess the distributions by fitting the observed durations to a set of well-known time distributions typically used in survival analysis. We consider the exponential, the Weibull, the Gamma and the log-normal distributions and fit them to the time spent in dependence. To decide which distribution yields the best fit, we use the Akaike information criterion (AIC). This criterion considers the likelihood function of the fitting and penalizes for higher number of parameters that need to be estimated. Lower AIC values indicate the better model, i.e. the better fitting distribution (Sakamoto et al., 1986). We report the AIC values in Table 5. Based on the numerical results, the Gamma distribution appears to be the closest to the empirical distribution for all care durations in both datasets. In fact, in all cases fitting a Gamma distribution leads to the smallest AIC value when compared to the other distributions. Thus, in our econometric study, we will assume Gamma-distributed LTC durations.

\begin{tabular}{|c|c|c|c|c|c|c|}
\hline \multirow[b]{2}{*}{ Distribution } & \multicolumn{3}{|c|}{$\mathcal{D}_{1}$} & \multicolumn{3}{|c|}{$\mathcal{D}_{2}$} \\
\hline & $D$ & $D^{\mathrm{HC}}$ & $D^{\mathrm{IC}}$ & $D$ & $D^{\mathrm{HC}}$ & $D^{\mathrm{IC}}$ \\
\hline Log-normal & 1774241 & 68111 & 1733824 & 658264 & 46118 & 631497 \\
\hline Exponential & 1772430 & 68847 & 1732090 & 657465 & 46504 & 630833 \\
\hline Weibull & 1759287 & 68025 & 1719865 & 653841 & 46070 & 627683 \\
\hline Gamma & 1757512 & 67966 & 1717948 & 653249 & 46035 & 627028 \\
\hline
\end{tabular}

Table 5: AIC values for the distribution fit of the overall $D$, at-home $D^{\mathrm{HC}}$ and institutional $D^{\mathrm{IC}}$ care durations in the data $\mathcal{D}_{1}$ and $\mathcal{D}_{2}$.

Explanatory variables The gender $G E$, the linguistic region $L R$, the type of household composition $H H$, the acuity level at entry $A L$, the types of care received $T C$ and the nation- 
ality $N A$ enter our modeling as categorical variables (see Table 1) and we consider the most frequently observed value as baseline. For the continuous variables used, i.e. the age at entry in dependence $\left(A G, A G^{\mathrm{HC}}\right.$ and $A G^{\mathrm{I} C}$ ) as well as the pre-retirement income $S A$, we further investigate how to deal with them. By fitting the age at entry $A G$ against the overall care duration $D$, we empirically observe a clear linear relationship, i.e. that the time spent in dependence linearly increases with the age of the elderly becoming dependent. Further, considering to derive age classes from an evolutionary tree approach, we find that classes should be built around each single age. These observations motivate our choice to introduce $A G$ as a continuous variable in a linear model. With regard to the pre-retirement income we stick with the four classes built on the quartiles as laid out in Section 2.4. In fact, the four categories can serve as a proxy for different socio-economic classes when giving an interpretation of the results. Finally, motivated by the second research objective, we consider the continuous variable of the at-home care duration $D^{\mathrm{HC}}$ for explaining the institutional care duration $D^{\mathrm{IC}}$. We code the duration spent in at-home care by introducing a categorical variable $D H$. Therein, we consider the case where no at-home care has been received, i.e. 0 months, and five classes along the number of months spent in at-home care: $1-3,4-12,13-24,25-36$ and above 36 months. The class grouping durations from one to three months incorporates the three months corresponding to the usual waiting period in the LTC insurance field. The classes considering the amounts of one, two and three years are based on the usual benefit period found in prevailing LTC products (Milliman, 2017).

Model setup and MLE estimation We aim to apply survival analysis techniques to describe the LTC duration. For this purpose, we rely on AFT models to identify the variables correlating with the time spent in dependence (Wei, 1992). This methodology accounts for the effect of time dependent covariates on the LTC duration and overcomes the time independent assumption required under the classical Cox proportional hazard rate models (Tseng et al., 2005; Patel et al., 2006). In fact, although Cox models are most commonly used, we cannot apply them since the Cox proportional assumption is rejected for some of the variables. ${ }^{8}$ AFT models account for right-censoring by adding a censoring indicator to the likelihood function (see below). The resulting estimated regression parameters are robust even if there are neglected factors (Hougaard, 1994, Keiding et al., 1997, Lin, 1998, Swindell, 2008). They also have an intuitive and direct explanation for the effect on the duration (Collett, 2015). The approach allows choosing the parametric specification for the underlying dependent variable and significantly improves the model when the selected parametric form adequately mimics the empirical one (Orbe et al., 2002). AFT models have found applications in many areas of research including, e.g., for investigating the risk factors affecting Alzheimer's disease (Lee et al., 2017), for mortality projections (Cadena and Denuit, 2016) and for analyzing the incident duration on freeways, i.e. the time from the occurrence of an incident to road clearance (Junhua et al., 2013).

We calibrate the model parameters using maximum likelihood estimation (MLE). Based on Klein and Moeschberger (1997), the likelihood function $\mathcal{L}$ aggregates the individual contributions $f_{Y}\left(y_{i} \mid \mathbf{Z}\right)^{\delta_{i}} \cdot\left[1-F_{Y}\left(y_{i} \mid \mathbf{Z}\right)\right]^{1-\delta_{i}}$ where $i$ denotes the respective observation. The notations $f_{Y}$

\footnotetext{
${ }^{8}$ In our models we explain the care durations using the variables contained in both data $\mathcal{D}_{1}$ and $\mathcal{D}_{2}$. When considering the Schoenfeld residuals test we find that the Cox proportional hazard rate model is not applicable to our data (Therneau and Grambsch, 2000). In fact, in our data $\mathcal{D}_{1}$, we observe that the Cox proportional assumption is rejected for the age at entry, the gender, the linguistic region and the type of care. The assumption is rejected for the same variables in the data $\mathcal{D}_{2}$.
} 
and $F_{Y}$ stand for the density respectively the cumulative distribution function of the considered care duration. The subscript $Y$ relates to the considered duration, i.e. $D, D^{\mathrm{HC}}$ resp. $D^{\mathrm{I} C}$. The functions $f_{Y}$ and $F_{Y}$ depend on the observations $y_{i}=D_{i}, D_{i}^{\mathrm{HC}}$ or $D_{i}^{\mathrm{IC}}$ and a set of covariates $\mathbf{Z}$. In the likelihood function, right-censored observations are accounted for by the indicator $\delta_{i}$ which is equal to zero when the record $i$ is censored. We have $\delta_{i}=1$ for uncensored observations. The function $\mathcal{L}$ writes out as follows:

$$
\mathcal{L}=\prod_{i} f_{Y}\left(y_{i} \mid \mathbf{Z}\right)^{\delta_{i}} \cdot\left[1-F_{Y}\left(y_{i} \mid \mathbf{Z}\right)\right]^{1-\delta_{i}}
$$

In our computations we use the $\log$-likelihood function $\ell=\log \mathcal{L}$, i.e. the logarithm of the likelihood function $\mathcal{L}$. We apply the $\mathrm{R}$ package 'flexsurv' to estimate the model parameters (Jackson, 2016).

\subsection{Specification of the regression models}

In the following, we develop three AFT models for explaining the care durations and apply them on both data $\mathcal{D}_{1}$ and $\mathcal{D}_{2}$. Our first model analyzes the overall duration $D$ (Equation 2) while the two others focus on the time spent while being cared for at home $D^{\mathrm{HC}}$ respectively in an institution $D^{\mathrm{IC}}$ (Equations 3 and 4). As argued above, we use a Gamma distribution for the durations and select the log-link specification as link function in the regression models. In the sequel, we follow the notation proposed by Lambert et al. (2004) and Swindell (2008) for AFT models.

From the available variables in $\mathcal{D}_{1}$ (see Table 1), we select those that help to best model the observed data using the stepwise forward selection approach (Venables and Ripley, 2002). We start with a constant, then we include single variables and finally consider combinations of all variables with the aim of finding the model returning the lowest AIC. Our selection process evidences that the overall duration $D$ is best explained when including the age, the gender, the linguistic region, the acuity level and the type of care, but disregarding the type of household. In the case of the duration of care received at home $D^{\mathrm{HC}}$, the type of household variable is relevant but we do not include the acuity level and the type of care variables. For the time spent in institutional care $D^{\mathrm{IC}}$, the selection process results in eliminating again the type of household from the econometric model and in including the categorical variable on the duration of care received at home. When applying the same procedure on the dataset $\mathcal{D}_{2}$, we come to the identical conclusion concerning the variables that enter into the model. With regard to the additional covariates available in the data $\mathcal{D}_{2}$, we observe that the pre-retirement income information yields relevant while the nationality variable does not appear in any model. In Table 6, we summarize the variables included in the models. In all models we aim to control for the heterogeneity from the calendar years, i.e. when the observations have been made. Thus, we include a term related to the fixed effects from years.

The model we use for explaining the overall care duration $D$, considering both the time spent in at-home and in institutional care, writes out as follows:

$$
\begin{aligned}
\log \left(D_{i}\right)= & \alpha+\beta_{A G} A G_{i}+\beta_{G E} G E_{i}+\beta_{L R} L R_{i}+\beta_{A L} A L_{i}+\beta_{T C} T C_{i}+\gamma+\epsilon_{i} \\
& \left(+\beta_{S A} S A_{i}\right) .
\end{aligned}
$$




\begin{tabular}{|c|c|c|c|c|c|c|c|c|c|c|}
\hline \multirow{2}{*}{\multicolumn{2}{|c|}{ Model }} & \multicolumn{7}{|c|}{$\mathcal{D}_{1}$ and $\mathcal{D}_{2}$} & \multicolumn{2}{|c|}{$\mathcal{D}_{2}$ only } \\
\hline & & $A G_{i}$ & $G E_{i}$ & $L R_{i}$ & $H H_{i}$ & $A L_{i}$ & $T C_{i}$ & $D H_{i}$ & $S A_{i}$ & $N A_{i}$ \\
\hline (2) & $D$ & $\checkmark$ & $\checkmark$ & $\checkmark$ & & $\checkmark$ & $\checkmark$ & & $\checkmark$ & \\
\hline (3) & $D^{\mathrm{HC}}$ & $\checkmark$ & $\checkmark$ & $\checkmark$ & $\checkmark$ & & & & $\checkmark$ & \\
\hline (4) & $D^{\mathrm{IC}}$ & $\checkmark$ & $\checkmark$ & $\checkmark$ & & $\checkmark$ & & $\checkmark$ & $\checkmark$ & \\
\hline
\end{tabular}

Table 6: Overview of the variables included in the econometric models (2) to (4).

The coefficients $\alpha, \gamma$ and $\epsilon_{i}$ stand for the intercept, the year fixed effects respectively the error term of the observation $i$. The coefficient $\alpha$ is related to the shape $\sigma$ and scale $\theta$ parameters of the Gamma distribution fitting the duration $D$ through $\alpha=\log (\sigma / \theta)$. The different $\beta$ are the regression coefficients linked to the variables age at entry $A G$, gender $G E$, linguistic region $L R$, acuity level at entry $A L$, type of care $T C$ and pre-retirement income $S A$, respectively. In the above equation, the term linked to the variable $S A$ appears in brackets since it is only used when applying the model on the data $\mathcal{D}_{2}$.

Using the same notations, we develop the econometric models for the duration of care received at home $D^{\mathrm{HC}}$,

$$
\begin{aligned}
& \log \left(D_{i}^{\mathrm{HC}}\right)=\alpha+\beta_{A G^{\mathrm{HC}}} A G_{i}^{\mathrm{HC}}+\beta_{G E} G E_{i}+\beta_{L R} L R_{i}+\beta_{H H} H H_{i}+\gamma+\epsilon_{i} \\
& \left(+\beta_{S A} S A_{i}\right) \text {, }
\end{aligned}
$$

and for the duration of care received in an institution $D^{\mathrm{IC}}$ :

$$
\begin{aligned}
\log \left(D_{i}^{\mathrm{I} C}\right)= & \alpha+\beta_{A G^{\mathrm{IC}}} A G_{i}^{\mathrm{I} C}+\beta_{G E} G E_{i}+\beta_{L R} L R_{i}+\beta_{A L} A L_{i}+\beta_{D H} D H_{i}+\gamma+\epsilon_{i} \\
& \left(+\beta_{S A} S A_{i}\right) .
\end{aligned}
$$

When compared to the model defined in Equation (2), both models (3) and (4) use the ages $A G^{\mathrm{HC}}$ and $A G^{\mathrm{IC}}$ specifically related to the moment when the elderly started being cared for at home respectively in an institution. In model (3) the type of household variable $H H$ is included, while in the last model (4), the variable $D H$ relates to the previous duration spent in at-home care.

\section{Results and discussion}

In the following, we address the three research objectives as follows: In Section 4.1, we apply the econometric models on our data and report on the impact of socio-economic factors on the LTC duration in Tables 7 and 8. Then, we present our findings on the interaction effect of at-home and institutional care on the overall care duration (Section 4.2). Finally, in Section 4.3, we lay out how the age at entry and the care duration have developed over the past years.

\subsection{Influence of socio-economic factors on the care duration}

In Tables 7 and 8, we present the results when applying models (2) to (4) to the data $\mathcal{D}_{1}$ respectively $\mathcal{D}_{2}$. For each model we report the estimates of the coefficients related to the various variables. To assess the statistical significance levels, we use "." for denoting a $p$-value lower than 0.1 and "** when it is below to 0.05 . The notations "*** and "**** stand for $p$ - 
values lower than 0.01 and 0.001 , respectively. We also give the shape $\sigma$ and scale $\theta$ parameters (together with the standard errors) of the Gamma distribution fitting the duration and recall the number of observations underlying each regression analysis (cf. also Table 4). ${ }^{9}$

Overall care duration We now highlight selected regression results for the overall care duration $D$ laid out in the first column (model 2) of Table 7 . We observe a negative effect of the age at entry on the duration $D$ with $\beta_{A G}=-0.039$. In fact, and as mentioned earlier, the time spent in LTC decreases at higher ages due to the mortality increase. Similarly, when comparing males to females, the males' gender coefficient is highly significant and negative $\left(\beta_{G E}=-0.293\right)$. At the same age, men remain less time in dependence than women. This outcome follows Mathers (1996), Mathers et al. (2001), Monod-Zorzi et al. (2007) and Fong et al. (2017) that explain such result by the higher mortality of men when compared to women. Further, women are more likely than men to live alone and therefore enter dependence only at higher ages (Schwarzkopf et al., 2012). Finally, the study of gender-specific prevalence of pathologies can infer that women present higher chances than men to develop Alzheimer diseases yielding to longer stays in LTC (Viña and Lloret, 2010; Colombo et al., 2011). The LTC duration decreases with higher acuity levels as also found by Rashwan et al. (2013) and Juma et al. (2016). In fact, a mildly dependent elderly exhibits a positive coefficient $\beta_{A L}$ of 0.500 while the severely dependents show a negative value $(-0.203)$ when compared to the moderately dependent ones. Our regression results highlight a strong effect of at-home and institutional care on the overall duration. Elderly receiving only at-home care stay more time in dependence $\left(\beta_{T C}=0.147\right)$ than dependent elderly having only received institutional care. Further, receiving both at-home and institutional care $\left(\beta_{T C}=-0.175\right)$ shows lower overall durations. This finding links to our below discussion of the interaction between at-home and institutional care.

The values of the coefficients $\beta_{L R}$ linked to the linguistic regions yield positive significant values for the French- and Italian-speaking regions (0.085 and 0.290). This means that, they stay longer in dependence when compared to elderly located in the German-speaking region. Such result suggests that the care pattern is affected by the place of residence. First, the longer stay can be due to cultural differences linked to the language regions. In fact, Gentili et al. (2017) and Eugster et al. (2011) find significant differences in both LTC and Swiss social insurance usage throughout the linguistic regions. Second, these differences can originate from the political orientation of Swiss cantons promoting different types of care (Dutoit et al., 2016). For example, cantons located in the French- and the Italian-speaking areas often favor at-home care for lighter cases of dependence and encourage to treat more complex cases in an institution. Such behavioral aspects result in individuals receiving LTC allowances at lower acuity levels and expanding their overall length of stay in dependence. Regional differences may also be the outcome of other unobserved factors such as cognitive diseases which often entail longer stays in dependence (Lafortune and Balestat, 2007; Monod-Zorzi et al., 2007). For example, the work of Chammartin et al. (2016) establishes a link between the place of residence and an individual's pathology profile. The authors find that Alzheimer prevalence rates are higher in the Frenchand in the Italian-speaking language regions.

\footnotetext{
${ }^{9}$ Although we have found that several covariates used in our models do not meet the Cox proportional assumption, see Footnote 8, it is worthwhile to review the fit by comparing the AIC values of the AFT and Cox proportional hazard rate approaches. In Table 13 in the Appendix, we report the AIC for both approaches and the models presented in Tables 7 and 8. Expectedly, we find that the AFT model outperforms the Cox approach.
} 


\begin{tabular}{|c|c|c|c|c|}
\hline \multirow[t]{2}{*}{ Model } & $(2)$ & (3) & \multicolumn{2}{|l|}{$(4)$} \\
\hline & $D$ & $D^{\mathrm{HC}}$ & \multicolumn{2}{|l|}{$D^{\mathrm{IC}}$} \\
\hline Age at entry & $-0.039(.000) * * *$ & $-0.038(.001) * * *$ & $-0.038(.000)$ & $* * *$ \\
\hline \multicolumn{5}{|c|}{ Gender (baseline: Female) } \\
\hline Male & $-0.293(.004) * * *$ & $-0.311(.021) * * *$ & $-0.285(.004)$ & $* * *$ \\
\hline \multicolumn{5}{|c|}{ Linguistic region (baseline: German) } \\
\hline French & $0.085(.004) * * *$ & $0.334(.023) * * *$ & $0.080(.004)$ & $* * *$ \\
\hline Italian & $0.290(.007) * * *$ & $0.088(.047)$ & $0.279(.007)$ & $* * *$ \\
\hline \multicolumn{5}{|c|}{ Type of household (baseline: Single person) } \\
\hline Two persons & & $-0.199(.021) * * *$ & & \\
\hline \multicolumn{5}{|c|}{ Acuity level at entry (baseline: Moderate) } \\
\hline Mild & $0.500(.048) * * *$ & & $0.566(.059)$ & $* * *$ \\
\hline Severe & $-0.203(.004) * * *$ & & $-0.203(.004)$ & $* * *$ \\
\hline \multicolumn{5}{|c|}{ Type of care (baseline: IC only) } \\
\hline $\mathrm{HC}$ only & $0.147(.048) * *$ & & & \\
\hline $\mathrm{HC}$ and IC & $-0.175(.049) * * *$ & & & \\
\hline \multicolumn{5}{|c|}{ Duration of care at home (baseline: 0 months) } \\
\hline $1-3$ months & & & $-0.733(.062)$ & $* * *$ \\
\hline $4-12$ months & & & $-0.815(.064)$ & $* * *$ \\
\hline $13-24$ months & & & $-0.830(.064)$ & $* * *$ \\
\hline $25-36$ months & & & $-0.772(.072)$ & *** \\
\hline Over 36 months & & & $-0.743(.083)$ & $* * *$ \\
\hline Shape $\sigma$ & $1.580(.005)$ & $1.057(.012)$ & $1.580(.005)$ & \\
\hline Scale $\theta$ & $0.001(.000)$ & $0.001(.000)$ & $0.001(.000)$ & \\
\hline Year fixed effect & Yes & Yes & Yes & \\
\hline$N$ total & 229117 & 20069 & 216520 & \\
\hline
\end{tabular}

Note: Significance levels are reported as follows: $p$-value $<0.1 .,<0.05^{*},<0.01 * *,<0.001 * * *$.

Table 7: Results for regression models (2) to (4) applied to the data $\mathcal{D}_{1}$.

At-home care duration Moving to the results from the regression model (3), we observe similar effects of the age at entry and the gender on the home care duration $D^{\mathrm{HC}}$ when compared with those on the overall duration $D$. In the sequel, we comment the main differences. First, for the linguistic regions, we observe a higher positive value of the French linguistic region coefficient (0.334) while the coefficient for the Italian region is not highly significant anymore. These differences can be interpreted along the lines of reasoning outlined above regarding regional policies. Second, we find a significant effect of the household composition on the at-home care duration. Being two-person reduces the duration of at-home care in comparison to elderly living in single households $\left(\beta_{H H}=-0.199\right)$. Our outcome is in line with the findings of Freedman and Martin (1998) which stipulates that elderly living in two-person household usually show functional limitations only at high ages when compared to single person household. In such context, mild dependence appearing most commonly along with at-home care is hidden by the help provided by the partner. Further, informal caregivers, not included in our data, strongly influence the formal LTC demand by providing themselves some extent of primary care (Courbage et al., 2018). 
Institutional care duration Outcomes from analyzing the institutional care duration $D^{\mathrm{IC}}$ are close to those from the study of the overall care duration $D$. This is not surprising since most individuals in our data have experienced institutional care (216 520 out of 229117 records). The coefficients values of the age at entry, the gender, the linguistic region and the acuity level at entry are quasi identical in both models (2) and (4). An interesting outcome of our study lies in the analysis of the reception of care at home before entering institutional care services. This is specifically addressed by the second resech objective presented in Section 4.2.

Results from the data $\mathcal{D}_{2}$ Following the same approach, we discuss the results obtained when applying the econometric models $(2)$ to (4) to the data $\mathcal{D}_{2}$ including income and nationality information. As mentioned earlier (see Section 3.2), the nationality variable does not enter any model since it does not add significant explanation to the model. We lay out the results in Table 8 . We observe that the results are very close to the findings reported in Table 7

\begin{tabular}{|c|c|c|c|c|c|c|}
\hline \multirow[t]{2}{*}{ Models } & (2) & \multicolumn{3}{|c|}{$(3)$} & \multicolumn{2}{|l|}{$(4)$} \\
\hline & $D$ & \multicolumn{3}{|c|}{$D^{\mathrm{HC}}$} & \multicolumn{2}{|l|}{$D^{\mathrm{IC}}$} \\
\hline Age at entry & $-0.038(.001) * * *$ & -0.033 & $(.002)$ & $* * *$ & $-0.039(.001)$ & $* * *$ \\
\hline \multicolumn{7}{|c|}{ Gender (baseline: Female) } \\
\hline Male & $-0.259(.008) * * *$ & -0.388 & $(.033)$ & $* * *$ & $-0.251(.008)$ & $* * *$ \\
\hline \multicolumn{7}{|c|}{ Linguistic region (baseline: German) } \\
\hline French & $0.094(.008) * * *$ & 0.376 & $(.029)$ & $* * *$ & $0.091(.008)$ & $* * *$ \\
\hline Italian & $0.282(.014) * * *$ & 0.074 & $(.061)$ & & $0.284(.014)$ & $* * *$ \\
\hline \multicolumn{7}{|c|}{ Type of household (baseline: Single person) } \\
\hline Two persons & & -0.233 & $(.027)$ & $* * *$ & & \\
\hline \multicolumn{7}{|c|}{ Acuity level (baseline: Moderate) } \\
\hline Mild & $0.439(.054) * * *$ & & & & $0.545(.068)$ & $* * *$ \\
\hline Severe & $-0.203(.007) * * *$ & & & & $-0.203(.068)$ & $* * *$ \\
\hline \multicolumn{7}{|c|}{ Type of care (baseline: IC only) } \\
\hline HC only & $0.255(.007) * * *$ & & & & & \\
\hline $\mathrm{HC}$ and $\mathrm{IC}$ & $-0.134(.055) * * *$ & & & & & \\
\hline \multicolumn{7}{|c|}{ Duration of care at home (baseline: 0 months) } \\
\hline $1-3$ months & & & & & $-0.723(.072)$ & $* * *$ \\
\hline $4-12$ months & & & & & $-0.840(.075)$ & $* * *$ \\
\hline $13-24$ months & & & & & $-0.756(.076)$ & $* * *$ \\
\hline $25-36$ months & & & & & $-0.759(.088)$ & $* * *$ \\
\hline Over 36 months & & & & & $-0.695(.102)$ & $* * *$ \\
\hline \multicolumn{7}{|c|}{ Pre-retirement income (baseline: $49539-77134$ ) } \\
\hline Below 22308 & $-0.024(.010) *$ & 0.026 & $(0.037)$ & & $-0.028(.010)$ & $* *$ \\
\hline $22308-49538$ & $0.039(.009) * * *$ & -0.028 & $(0.035)$ & & $0.039(.010)$ & $* * *$ \\
\hline Over 77134 & $-0.034(.009) * * *$ & 0.033 & $(0.037)$ & & $-0.035(.010)$ & $* * *$ \\
\hline Shape $\sigma$ & $1.490(.008)$ & 1.013 & $(.015)$ & & $1.460(.008)$ & \\
\hline Scale $\theta$ & $0.001(.000)$ & 0.002 & $(.002)$ & & $0.001(.000)$ & \\
\hline Year fixed effect & Yes & & Yes & & Yes & \\
\hline$N$ total & 92898 & & 3871 & & 84011 & \\
\hline
\end{tabular}

Note: Significance levels are reported as follows: $p$-value $<0.1 .,<0.05^{*},<0.01 * *,<0.001 * * *$.

Table 8: Results for regression models (2) to (4) applied to the data $\mathcal{D}_{2}$. 
using data $\mathcal{D}_{1}$. This confirms that the smaller size of $\mathcal{D}_{2}$ and the differences in the composition of $\mathcal{D}_{2}$ when compared to $\mathcal{D}_{1}$ (e.g., more males and higher ages) have no relevant influence on the final outcome. The inclusion of the pre-retirement income information brings significant evidence on its interaction with the time spent in dependence. In the reporting of our results, we select the category CHF 49539 - 77134 as baseline. In the case of the overall care duration $D$ (regression model 2), we note a significantly higher time spent in dependence for pre-retirement incomes in the group $22308-49538\left(\beta_{S A}=0.039\right)$ while we find a significantly lower overall care duration for incomes above $77134\left(\beta_{S A}=-0.034\right)$ when compared to the baseline. Elderly with higher pre-retirement income tend to spend less time in LTC dependence. This corroborates with the results of Brown and Finkelstein (2009) highlighting that wealthier individuals are usually more informed about their future long-term care needs and are less likely to be in dependence (see also, Mcgarry, 2011). Indeed, the study of Fried et al. (2001) shows that wealthier individuals report less limitations in ADL. This is also confirmed by Mobley et al. (2006) where the authors explain such effect by the benefits from higher care all along their life and the supplemental coverage healthier individuals hold. In addition, the latter are often homeowners who can facilitate cohabitation with relatives, simplify the installation of equipment for the disabled and thus foster at-home care use (Rodrigues et al., 2018). The results found for the institutional care duration lead to the same conclusion. The estimated pre-retirement income coefficients for the at-home care duration do not yield significant findings.

\subsection{Interaction effect of types of care}

In the two graphs of Figure 3, we report the median overall care duration $m_{D}$ and the median institutional care duration $m_{D^{\mathrm{IC}}}$ along the observed time $D^{\mathrm{HC}}$ an individual has spent in athome care. Both graphs are based on the 216520 elderly recorded in $\mathcal{D}_{1}$ that have been cared for in an institution and that have or have not received at-home care before (see column $D^{\mathrm{IC}}$ in Table 4). From the 7472 records (see also Section 2.2) that have experienced both at-home and institutional care, 1544 observations relate to at-home care durations above two years. In Figure 3, we limit our illustration to individuals that have received up to 24 months of at-home care. This leaves us with 209048 observations where no at-home care has been delivered before institutional care and 5928 observations where both at-home and institutional care have been provided.

From Figure 3a, we observe an overall median duration $m_{D}$ of about 32 months when no athome care is received (black dot). The plain segments reflect the value of the median overall care duration $m_{D}$ for a given group of at-home care durations $D^{\mathrm{HC}}$ (1 to 6,7 to 12,13 to 18 and 19 to 24 months). For each group we provide the $95 \%$ confidence interval. Due to the important share of right-censoring, the upper confidence bound cannot be evaluated in the last group (19 to 24 months). A dashed line starting at $m_{D}=32$ months in the absence of at-home care (0 months) simulates the proportional increase of the overall duration when additionally at-home care is provided, i.e. each additional month in at-home care adds one month in the median overall care duration. Estimated median overall care durations $m_{D}$ along $D^{\mathrm{HC}}$ that lie below the dashed line indicate an under proportional increase between $m_{D}$ and $D^{\mathrm{HC}}$ indicating a beneficial effect of at-home care on the overall duration. Case where $m_{D}$ lies above the dashed line relate to an over proportional relation between $m_{D}$ and $D^{\mathrm{HC}}$. We note two patterns when at-home care is provided. First, if at-home care is provided between 1 and 6 months, the median overall care duration is 34 months. This median duration becomes 38 months for 7 to 12 


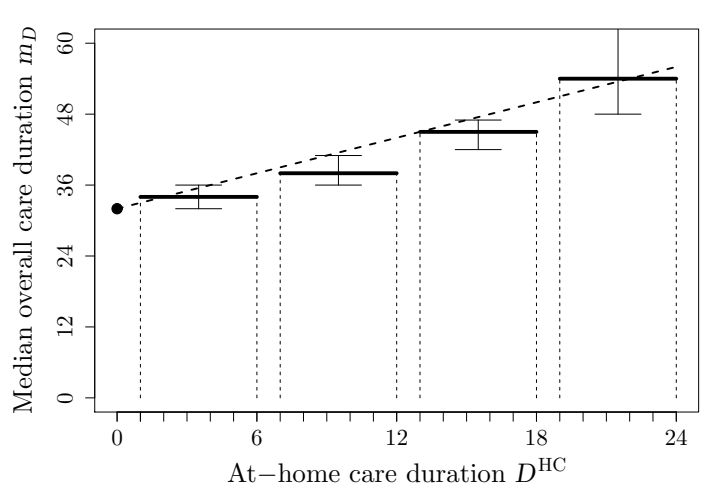

(a)

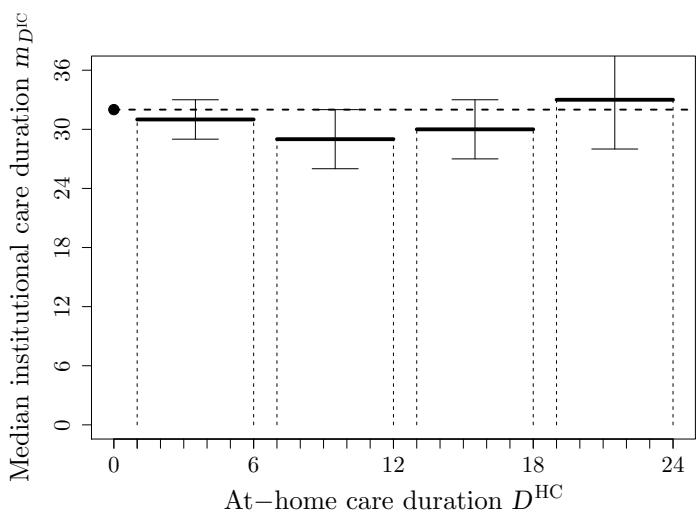

(b)

Note: Durations are expressed in months. Statistics are based on the data $\mathcal{D}_{1}$. Confidence intervals are given at the $95 \%$ level.

Figure 3: Effect of the at-home care duration $D^{\mathrm{HC}}$ on the median overall $\left(m_{D}\right)$ and institutional $\left(m_{D^{\text {IC }}}\right)$ care durations.

months of at-home care. For the latter, the upper bound of the confidence interval (centered on the $7-12$ interval, i.e. at 9.5 months) lies below the dashed line (41.5 months). We conclude that in this case providing at-home care for up to 12 months has a significant beneficial effect on the overall care duration, i.e. when providing at-home care before institutional care, the median overall care duration increases less than the sum of at-home and institutional care. This signals that the institutional care duration is reduced (see also the discussion below on graph b). Second, when providing more than 12 months of at-home care, the increase in the median overall care duration is important. For example, $m_{D}$ is 54 months when at-home care is provided for 18 to 24 months. Through the graph, we observe a trend leading to median overall care durations increasing proportionally and even over proportionally. Therefore, such outcome indicates a non-substitution effect with at-home care adding to institutional care.

In Figure 3b, the first reported median time (black dot) as well as the horizontal dashed line stand for individuals that have only received institutional care. The plain segments and the associated $95 \%$ confidence intervals relate to the estimated median institutional care duration $m_{D^{\mathrm{IC}}}$ for groups of at-home care durations $D^{\mathrm{HC}}$. Looking at the reported values, we observe that the median duration $m_{D^{\mathrm{IC}}}$ is about 32 months when the elderly has not previously received at-home care. When receiving between 1 and 6 months of at-home care, $m_{D^{\text {IC }}}$ decreases to 31 months. It reduces to 29 months when the at-home care duration lies between 6 and 12 months. Therefore, delivering up to 12 months of at-home care leads to a reduction in the duration of institutional care. However, such change is significant only for at-home care durations between 6 and 12 months (cf. the upper bound of the confidence interval below the dashed line). At-home care durations between 12 and 24 months lead to a median institutional care duration close to 30 months indicating further irreducibility of institutional care. This empirical result highlights a non-reducible amount of institutional care that certainly deserves further investigation in another context. ${ }^{10}$ In the sequel, we deepen our analysis using the results reported in Sec-

\footnotetext{
${ }^{10}$ To go further, we present the numerical values of graph (b) in Table 14 in the Appendix and study the significance of our findings. We perform log-rank tests to compare the Kaplan-Meier estimates of the survival curves for the institutional care duration. In each test, we consider one of the four groups of at-home care
} 
tion 4.1 .

From the results on the institutional care duration $D^{\mathrm{IC}}$ (regression model 4) presented in Table 7 , we observe that elderly having received at-home care prior to care in an institution experience significantly less time in institutional care than those having not received care at home before (0 months). For all at-home care duration categories, the coefficients yield highly significantly negative values. In Figure 4, we separately lay out the coefficient values reported in Table 7 and include information on the $95 \%$ and $90 \%$ confidence intervals. For 1 to 3 months of at-home care we have $\beta_{D H}=-0.733$. At the $90 \%$-confidence level, the coefficients for durations of 4 to 12 months $\left(\beta_{D H}=-0.815\right)$ and of 13 to 24 months $\left(\beta_{D H}=-0.830\right)$ statistically diverge from the one found for durations of 1 to 3 months. Their relative difference is not strong enough to become evidence at the $95 \%$ confidence level (see Figure 4 ). For at-home care durations from 25 to 36 months $\left(\beta_{D H}=-0.772\right)$ and over 36 months $\left(\beta_{D H}=-0.743\right)$, we find again higher values.

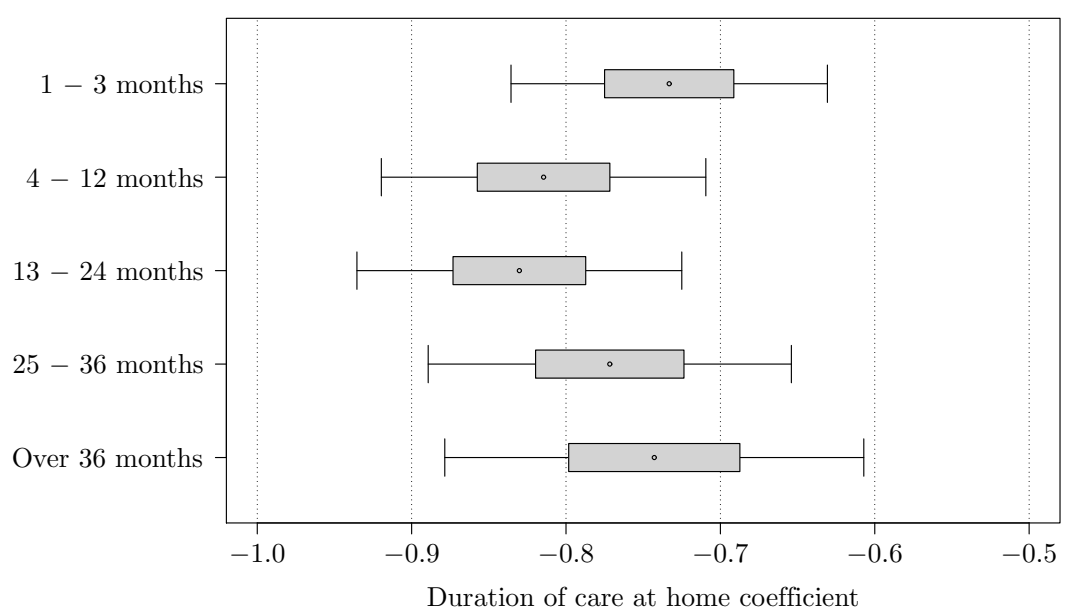

Note: Confidence intervals are given at the $90 \%$ (gray shaded bars) and $95 \%$ levels (solid lines).

Figure 4: Illustration of the effect $\left(\beta_{\mathrm{DH}}\right)$ of selected at-home care durations $D H$ on the institutional care duration $D^{\mathrm{IC}}$ in model (4) applied to the data $\mathcal{D}_{1}$.

Our findings highlight the co-existence of both substitution and non-substitution effects between the two types of care. In fact, the importance of the negative effect of receiving at-home care on the duration $\mathcal{D}^{\mathrm{IC}}$ indicates substitution. A significant part of the institutionalized care can be reduced by prior at-home care and this lay the basis for encouraging local policies fostering LTC at-home usage. However, when the time spent at home has been longer, i.e. above 12 months, any increase of the at-home care duration does not lead to a significant decrease of the institutional LTC duration hinting a non-substitution effect. Such outcome raises something we can call non-reducible part of institutional care. In fact, there exist a multitude of factors impeding complete substitution of institutional care by home care. For example, individuals diagnosed with dementia have to be cared for in an institution after reaching a

durations $D^{\mathrm{HC}}$ and compare the related Kaplan-Meier estimate with the estimate when no at-home care is received (baseline, $D^{\mathrm{HC}}=0$ ). We find that, although, in graph (b), the $95 \%$ confidence intervals overlap the horizontal dashed line for most at-home care durations, the log-rank test reports significant differences between the Kaplan-Meier estimates related to the baseline $\left(D^{\mathrm{HC}}=0\right)$ and the at-home care durations groups of $7-12$ and $13-18$ months. 
high stage of dependence due to the need of permanent supervision, highly qualified medical staff or specialized infrastructure (Callahan et al., 2012). Nonetheless, we also need to be very careful when commenting on the observed beneficial substitution effect because our data mostly contains paths of dependent persons only observed after entry into care and ending with death. While, on the one hand, postponing death during ill health entails higher costs, on the other hand, reducing the time spent in dependence means dying earlier.

\subsection{Evolution of the age at entry and the time spent in dependence}

Using statistical analyses, we assess the development of the average age when elderly become dependent and the changes of the time spent in dependence over the past years. We base our analysis on the records from the data $\mathcal{D}_{1}$ and consider all elderly that entered in dependence within the calendar years between 1995 and 2009. For the present analysis we apply this particular restriction since, from 2010 onwards the share of right-censored data strongly increases and exceeds half of the observations after 2013 (cf. Table 3 in Section 2.3). Although the application of the Kaplan-Meier estimation method is always appropriate for descriptive statistics, its use for providing statistical evidence on the duration analysis leads to severe bias when the share of right-censored data is high (Karadeniz and Ercan, 2017).

Age at entry For studying the old-age dependence entry age, we compare the mean age at entry by gender and by year from our data with statistics from the FSO. The FSO reports that at the age of 80 years, an age that is representative for the mean age of dependent elderly, the male life expectancy was 7.1 years in 1995 and 8.4 years in 2009. For females at same age, the values are 8.9 years and 10 years in 1995 and 2009, respectively. This indicates a gain in life expectancy of slightly more than one year for both genders. In Table 9 we display the mean age at entry in dependence (column " $\varnothing A G$ ") for calendar years from 1995 to 2009 and both genders using the data $\mathcal{D}_{1}$. We present the $5 \%$ - $\left(q_{5 \%}\right)$ and $95 \%$-quantiles $\left(q_{95 \%}\right)$, as well as the number $N$ of records for each calendar year and gender combination. For the year 1995, we find a mean age at entry of 81.3 years for men and 84.9 years for women. In 2009, these values yield 82.2 years and 85.7 years, respectively. This reveals an increase of about 0.9 years and 0.8 years for males respectively females within the 15 -year period. The same changes appear in the males' 95\%-quantile and the females' $5 \%$ - and 95\%-quantiles which undergo a one year increase over the period of observation. The row labeled "'95 vs '09" reports the significance level of a $t$-test comparing the age at entry in dependence in 1995 and 2009. Such statistical approach is valid

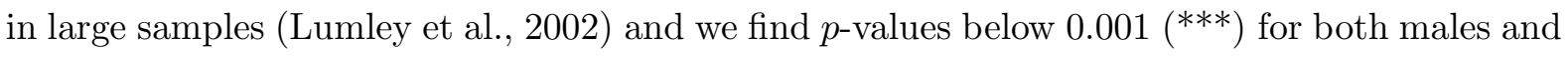
females ensuring significant differences in the compared mean ages. From the aforementioned findings, we conclude that the age at entry in dependence has shifted towards higher ages in line with the longevity improvement.

Care duration In the following, we discuss changes in the overall time spent in dependence that appear over the years. In Table 10, we present the evolution of the median overall duration resulting from the Kaplan-Meier estimates. For both genders and for the ages of 70, 80 and 90 years, we report the median time in dependence $\left(m_{D}\right)$ for the calendar years from 1995 to 2009. Our methodology consists in comparing Kaplan-Meier estimates on the records of $\mathcal{D}_{1}$ clustered by the year of entry in dependence, by the age at entry and by the gender. We assess the significance of changes in the median overall care duration $m_{D}$ by applying the Peto \& Peto modification of the Gehan-Wilcoxon test (Harrington and Fleming, 1982). Thereby, we value 


\begin{tabular}{|c|c|c|c|c|c|c|c|c|}
\hline \multirow{2}{*}{$\begin{array}{c}\text { Calendar } \\
\text { year }\end{array}$} & \multicolumn{4}{|c|}{ Male } & \multicolumn{4}{|c|}{ Female } \\
\hline & $\varnothing A G$ & $q_{5 \%}$ & $q_{95 \%}$ & $(N)$ & $\varnothing A G$ & $q_{5 \%}$ & $q_{95 \%}$ & $(N)$ \\
\hline 1995 & 81.3 & 69 & 93 & $(2510)$ & 84.9 & 72 & 95 & $(5778)$ \\
\hline 1996 & 81.4 & 69 & 92 & $(2514)$ & 85.0 & 72 & 95 & $(6007)$ \\
\hline 1997 & 81.4 & 68 & 93 & $(2831)$ & 85.2 & 72 & 95 & $(6366)$ \\
\hline 1998 & 81.5 & 69 & 93 & $(2859)$ & 85.1 & 73 & 95 & $(6454)$ \\
\hline 1999 & 81.3 & 69 & 93 & (3 016) & 85.3 & 73 & 95 & $(6507)$ \\
\hline 2000 & 81.7 & 68 & 93 & (3 208) & 85.2 & 72 & 95 & $(7156)$ \\
\hline 2001 & 81.7 & 69 & 93 & (3418) & 85.4 & 73 & 96 & $(7293)$ \\
\hline 2002 & 81.7 & 68 & 93 & $(3270)$ & 85.5 & 73 & 96 & $(7236)$ \\
\hline 2003 & 82.1 & 69 & 94 & $(3372)$ & 85.5 & 73 & 96 & $(7437)$ \\
\hline 2004 & 82.2 & 69 & 94 & $(3440)$ & 85.6 & 73 & 96 & $(7634)$ \\
\hline 2005 & 82.4 & 69 & 94 & (3439) & 85.6 & 73 & 96 & $(7569)$ \\
\hline 2006 & 82.4 & 69 & 94 & $(3433)$ & 85.6 & 73 & 96 & $(7286)$ \\
\hline 2007 & 82.6 & 69 & 94 & $(3500)$ & 85.7 & 73 & 96 & $(6994)$ \\
\hline 2008 & 82.2 & 69 & 94 & $(3460)$ & 85.7 & 73 & 96 & $(7114)$ \\
\hline 2009 & 82.2 & 68 & 95 & $(3711)$ & 85.7 & 73 & 96 & $(7437)$ \\
\hline '95 vs '09 & $* * *$ & & & & & & & \\
\hline
\end{tabular}

Note: Calendar years $(1995-2009)$ denote the respective year of entry in dependence. The column " $\varnothing A G$ " reports the mean age at entry. $q_{5 \%}$ and $q_{95 \%}$ denote the $5 \%$ - respectively $95 \%$-quantiles. The number of observations $N$ is given in parentheses. Statistics are based on the data $\mathcal{D}_{1}$. Significance levels between ' 95 and ' 09 are reported as follows: $p$-value $<0.001 * * *$.

Table 9: Evolution of the mean age at entry for male and female between 1995 and 2009.

the statistical significance of changes in the overall care duration across calendar years and between both genders for three selected ages. We decide to use such test instead of the log-rank test because, firstly, under a non-constant hazard ratio, the Peto \& Peto modification performs better than the log-rank test (Pepe and Fleming, 1989; Karadeniz and Ercan, 2017). Secondly, the test we rely on gives more weight to the observations of shorter durations in the distribution what is more suited for our analysis. This diverges from the constant weight approach used in the log-rank test. Our table informs about the number of observations $(N)$ underlying each estimate. The last three columns report the $\chi^{2}$-result for the different years and ages of entry in dependence testing the hypothesis that the care duration distributions across both genders are identical. Using a similar logic, the last row labeled "'95 - '09" reports the result from the significant differences test across the calendar years of entry.

The results for the males entering dependence at an age of 70 years exhibit a volatile median duration $m_{D}$ over the calendar years. We notice that those men who have entered dependence in 1995 show a median duration of 61 months. Such persons having entered in 2009 are characterized by $m_{D}=31$ months. For entries in dependence at the age of 70 years, the number of records $N$ in each calendar year is below one hundred. Given the higher number of observations for elderly that entered dependence at the ages of 80 and 90 years, we obtain more stable results. In fact, for 80 respectively 90 years old men, the median LTC duration is about 30 and 20 months. The same remarks regarding stability apply for female. Considering the numerical results, we observe that women entering dependence at an age of 80 and 90 years, receive, in the median, about 40 and 30 months of LTC, respectively. 


\begin{tabular}{|c|c|c|c|c|c|c|c|c|c|c|c|c|c|c|c|}
\hline \multirow{3}{*}{$\begin{array}{c}\text { Calendar } \\
\text { year }\end{array}$} & \multicolumn{6}{|c|}{ Male } & \multicolumn{6}{|c|}{ Female } & \multicolumn{3}{|c|}{$\mathrm{M} / \mathrm{F}$} \\
\hline & \multicolumn{2}{|c|}{70} & \multicolumn{2}{|c|}{80} & \multicolumn{2}{|c|}{90} & \multicolumn{2}{|c|}{70} & \multicolumn{2}{|c|}{80} & \multicolumn{2}{|c|}{90} & \multirow[t]{2}{*}{70} & \multirow[t]{2}{*}{80} & \multirow[t]{2}{*}{90} \\
\hline & $m_{D}$ & $(N)$ & $m_{D}$ & $(N)$ & $m_{D}$ & $(N)$ & $m_{D}$ & $(N)$ & $m_{D}$ & $(N)$ & $m_{D}$ & $(N)$ & & & \\
\hline 1995 & 61 & (49) & 30 & $(113)$ & 21 & $(89)$ & 67 & $(38)$ & 40 & $(214)$ & 27 & $(320)$ & & $* * *$ & $*$ \\
\hline 1996 & 49 & $(40)$ & 31 & $(106)$ & 21 & (99) & 55 & $(65)$ & 40 & $(174)$ & 28 & $(328)$ & & $* * *$ & $* *$ \\
\hline 1997 & 47 & (55) & 23 & (93) & 21 & (90) & 55 & $(38)$ & 42 & $(200)$ & 25 & $(340)$ & & $* * *$ & $*$ \\
\hline 1998 & 50 & (61) & 25 & $(135)$ & 19 & (83) & 59 & (41) & 40 & (199) & 28 & $(381)$ & & $* * *$ & $* * *$ \\
\hline 1999 & 33 & (72) & 30 & $(149)$ & 25 & (95) & 52 & (43) & 44 & $(206)$ & 29 & $(388)$ & & $* * *$ & $* *$ \\
\hline 2000 & 37 & (61) & 25 & $(152)$ & 26 & $(109)$ & 66 & $(52)$ & 43 & $(272)$ & 31 & $(410)$ & $*$ & $* * *$ & $*$ \\
\hline 2001 & 52 & (58) & 33 & $(164)$ & 20 & $(121)$ & 45 & $(45)$ & 45 & $(248)$ & 26 & $(426)$ & & $* * *$ & $* * *$ \\
\hline 2002 & 52 & (69) & 27 & $(156)$ & 21 & $(127)$ & 52 & $(39)$ & 42 & $(240)$ & 27 & $(447)$ & & $* * *$ & $* * *$ \\
\hline 2003 & 40 & (60) & 35 & $(146)$ & 24 & $(109)$ & 67 & (57) & 41 & $(267)$ & 29 & $(406)$ & $* *$ & * & $* * *$ \\
\hline 2004 & 36 & (68) & 28 & $(146)$ & 22 & (133) & 59 & (57) & 47 & $(262)$ & 26 & $(477)$ & $* *$ & $* * *$ & $*$ \\
\hline 2005 & 55 & (63) & 28 & $(164)$ & 21 & (123) & 80 & (53) & 41 & (269) & 29 & $(380)$ & $*$ & $* * *$ & $* *$ \\
\hline 2006 & 39 & $(50)$ & 36 & $(151)$ & 19 & $(114)$ & 60 & (58) & 41 & $(250)$ & 30 & $(333)$ & $* *$ & & $* * *$ \\
\hline 2007 & 52 & (39) & 34 & $(155)$ & 20 & (137) & 64 & $(45)$ & 44 & $(238)$ & 31 & $(333)$ & & $* * *$ & $* * *$ \\
\hline 2008 & 42 & $(56)$ & 33 & $(164)$ & 23 & $(128)$ & 83 & (44) & 44 & $(239)$ & 26 & $(368)$ & $* *$ & $* * *$ & $* * *$ \\
\hline 2009 & 31 & (71) & 31 & $(173)$ & 19 & $(119)$ & 50 & (44) & 43 & (168) & 30 & $(413)$ & $*$ & $* * *$ & $* * *$ \\
\hline '95 - '09 & & & & & & & & & & & & & & & \\
\hline
\end{tabular}

Note: Calendar years $(1995$ - 2009) denote the respective year of entry in dependence. Ages refer to the age at entry. Durations are expressed in months. The number of observations $N$ is given in parentheses. Statistics are based on the data $\mathcal{D}_{1}$. Significance levels between male and female $(\mathrm{M} / \mathrm{F})$ and across the calendar years ('95 - '09) are reported as follows: $p$-value $<0.10^{*},<0.05^{* *},<0.01^{* * *}$.

Table 10: Median overall care duration $m_{D}$ for male and female at the ages of 70,80 and 90 years between 1995 and 2009.

Considering the results of the statistical tests, we observe that the hypothesis of identical care duration distributions across both genders is rejected in many cases. On the one hand, for the age at entry in dependence of 70 years, statistical significance is observed in few calendar years only. On the other hand, at the ages of 80 and 90 years, the median durations are significantly different between both genders over most calendar years. While we mostly observe strongly significant results $(p$-values $<0.01)$, we evidence that women consistently show higher median overall care durations when compared to men. Our results confirm the importance of the gender as a major factor in econometric modeling (also see Section 3). Moving to the results of the statistical tests across the calendar years (see the bottom row of Table 10), we note that the tests on the care duration distribution identity cannot be rejected in all cases. In fact, only low significance $(p$-values $<0.10)$ supports the rejection of the hypothesis in the cases of male that entered dependence at an age of 80 years and of female at an age of 90 years. Based on these findings, we conclude that the median time spent in dependence has not significantly changed over the years, i.e. we do not support the theory of Fries (1980) and Olshansky et al. (1990) in the context of LTC. This answers part of research question 3 and we state that, based on our data, the duration of LTC has not significantly changed in the period from 1995 to 2009. In Figure 5, we illustrate the median overall care duration $m_{D}$ for the period from 1995 to 2009 . The two graphs report the median duration for both genders at the ages of entry in dependence of 80 respectively 90 years. We provide the $95 \%$ confidence interval illustrating the significant differences between both genders and the irrelevant changes of the duration over the calendar years. 


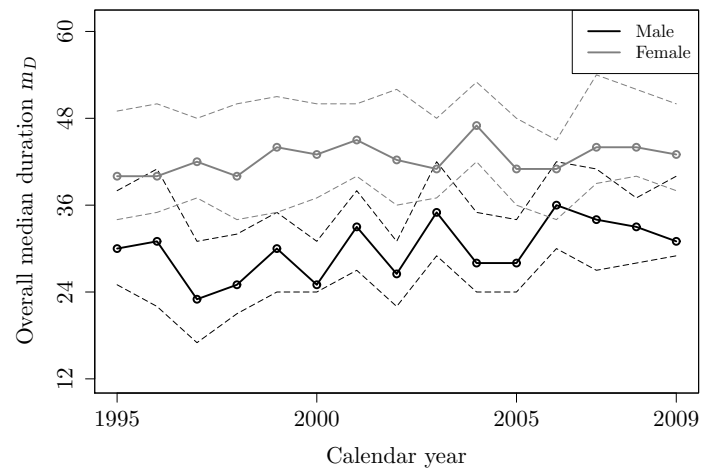

(a) Age 80

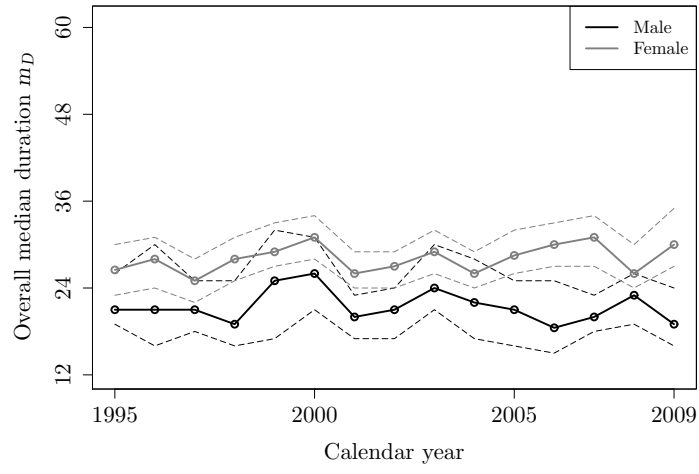

(b) Age 90

Note: Calendar years $(1995$ - 2009) denote the respective year of entry in dependence. Ages refer to the age at entry. Durations are expressed in months. Numerical values for the median duration and the number of observations are reported in Table 10. Statistics are based on the data $\mathcal{D}_{1}$. Dashed lines indicate the confidence interval at the $95 \%$ level.

Figure 5: Illustration of the median overall care duration $m_{D}$ for male and female between 1995 and 2009 at the ages of 80 and 90 years.

\section{Applications for insurers and policymakers}

In the following, we use the model developed and calibrated above to predict the time spent in dependence along different individual profiles (Section 5.1). Further, we discuss potential implications for insurers and policymakers in Section 5.2.

\subsection{Prediction for selected profiles}

Using the estimates from model (2) applied to the data $\mathcal{D}_{2}$ (cf. Table 8), we present in this section the predicted mean and median of the total care duration with the respective $95 \%$ confidence interval around the mean. Thereby, the mean and median correspond to the average respectively 50\%-quantile of the predicted survival curve from the AFT model (2). For both genders we consider the same reference profile ("Base") reflecting the most common characteristics recorded in our data. This base profile corresponds to 80 years old $(A G)$ elderly living in the German-speaking language region $(L R)$, entering dependence with a moderate acuity level $(A L)$ in 2011, receiving only institutional care $(T C)$ and having had a pre-retirement income $(S A)$ between CHF 49539 and 77134 . In Tables 11 and 12 we report prediction results for men respectively women and we consider several profiles (A to K) to illustrate the sensitivity. Ceteris paribus, we consider alternative profiles where at each time we change the value of one characteristic only: alternative ages at entry of 70 respectively 90 years, two other language regions (French and Italian), mild and moderate acuity levels at entry, different paths of types of care (at-home care only and the combination of both types of care) as well as pre-retirement incomes lying within the other three categories.

Analyzing first the prediction for the male profiles (see Table 11), the age at entry appears to be a major factor of influence. For the base profile, we predict a mean overall care duration of 44.0 months within a $95 \%$-confidence interval between 42.8 and 45.2 months. The predicted median time spent in care is evaluated at 34.8 months. These values can be compared to those 


\begin{tabular}{clllllllll}
\hline Profile & $A G$ & $L R$ & $A L$ & $T C$ & $S A$ & $\widehat{\varnothing D}$ & $\widehat{\varnothing \widehat{D l o w e r}_{\text {lor }}}$ & $\varnothing \widehat{D_{\text {upper }}}$ & $\widehat{m_{D}}$ \\
\hline Base & 80 & German & Moderate & IC & cat. 3 & 44.0 & 42.8 & 45.2 & 34.8 \\
\hline A & $\mathbf{7 0}$ & German & Moderate & IC & cat. 3 & 62.3 & 60.7 & 63.8 & 51.2 \\
B & $\mathbf{9 0}$ & German & Moderate & IC & cat. 3 & 30.1 & 29.3 & 31.0 & 23.7 \\
\hline C & 80 & French & Moderate & IC & cat. 3 & 48.2 & 46.9 & 49.4 & 38.3 \\
D & 80 & Italian & Moderate & IC & cat. 3 & 57.2 & 55.3 & 58.9 & 46.2 \\
\hline E & 80 & German & Mild & IC & cat. 3 & 64.7 & 59.1 & 70.1 & 53.7 \\
F & 80 & German & Severe & IC & cat. 3 & 36.1 & 35.2 & 37.1 & 28.4 \\
\hline G & 80 & German & Moderate & HC & cat. 3 & 56.0 & 51.1 & 61.4 & 45.1 \\
H & 80 & German & Moderate & HC and IC & cat. 3 & 38.8 & 34.2 & 43.1 & 30.6 \\
\hline I & 80 & German & Moderate & IC & cat. 1 & 43.0 & 42.0 & 44.1 & 34.0 \\
J & 80 & German & Moderate & IC & cat. 2 & 45.7 & 44.4 & 46.9 & 36.2 \\
K & 80 & German & Moderate & IC & cat. 4 & 42.6 & 41.5 & 43.6 & 33.6 \\
\hline
\end{tabular}

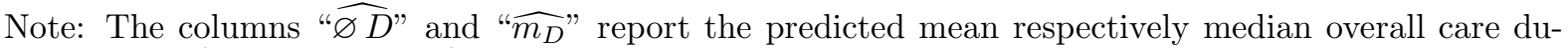

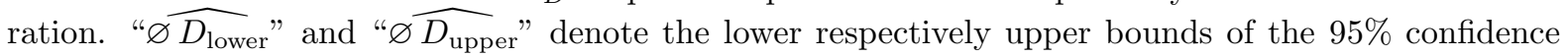
intervals on the predicted mean duration. Durations are expressed in months. The pre-retirement income categories $S A$ are as follows: "cat. 1" is below 22308 , "cat. 2" is $22308-49538$, "cat. 3" is $49539-$ 77134 and "cat. 4" is over 77134 . The gray shaded row refers to the reference profile.

Table 11: Predictions of the overall care duration for males along representative characteristics.

of elderly entering dependence at the ages of 70 and 90 years (profiles A and B) where we find mean durations of 62.3 and 30.1 months and median times of 51.2 and 23.7 months, respectively. The range of our results are in line with the findings from other studies showing that the average time in dependence for a 85 years old male is of about three years (cf. Monod-Zorzi et al., 2007, and Dutoit et al., 2016, who both neglect right-censoring). Indeed, we observe that a 36-month average lies between our 44.0 and 30.1 months estimates for the ages of 80 and 90 years, respectively. Important changes in our predictions arise also when looking at other linguistic regions of residence. Elderly along profile $\mathrm{C}$ living in the French-speaking region show a predicted mean duration of 48.2 months while those living in the Italian-speaking region (profile D) present a mean time of 57.2 months. Further, the most important variation in duration occurs when comparing elderly with different acuity levels at entry. The mean and median duration are of 64.7 and 53.7 months for a mildly dependent (profile E) while they are of 36.1 and 28.4 months for a severely dependent elderly (profile F). With regard to the types of care, on the one hand, having received only at-home care leads to an increase of 12 and 10 months in both the mean respectively the median duration. On the other hand, receiving both at-home and institutional care results in a decrease of the values. Finally, the comparison of the various pre-retirement income categories does not show important differences in the predicted durations.

When focusing on the results for women (Table 12), we observe the same patterns than for men with the exception that women stay much longer in dependence. In fact, we observe that females' mean durations exceed those from male by 12.0 months (56.0 vs. 44.0 months). As discussed in Section 4.1, other studies also found that females remain longer time in dependence than males (see, e.g., Monod-Zorzi et al., 2007) and can be explained by the lower female mortality at higher ages (Mathers et al., 2001; Fong et al., 2017). Finally, we observe again most important changes in the duration coming from changes in the age and acuity level at entry. 


\begin{tabular}{clllllllll}
\hline Profile & $A G$ & $L R$ & $A L$ & $T C$ & $S A$ & $\widehat{\varnothing D}$ & $\widehat{\varnothing \widehat{D l o w e r}_{\text {lor }}}$ & $\varnothing \widehat{D_{\text {upper }}}$ & $\widehat{m_{D}}$ \\
\hline Base & 80 & German & Moderate & IC & cat. 3 & 56.0 & 54.8 & 57.0 & 45.1 \\
\hline A & $\mathbf{7 0}$ & German & Moderate & IC & cat. 3 & 74.7 & 73.4 & 76.0 & 66.3 \\
B & $\mathbf{9 0}$ & German & Moderate & IC & cat. 3 & 39.0 & 38.0 & 40.1 & 30.7 \\
\hline C & 80 & French & Moderate & IC & cat. 3 & 60.7 & 59.3 & 62.2 & 49.6 \\
D & 80 & Italian & Moderate & IC & cat. 3 & 70.0 & 68.4 & 71.8 & 59.8 \\
\hline E & 80 & German & Mild & IC & cat. 3 & 76.8 & 71.9 & 80.8 & 69.6 \\
F & 80 & German & Severe & IC & cat. 3 & 46.5 & 45.3 & 47.7 & 36.9 \\
\hline G & 80 & German & Moderate & HC & cat. 3 & 68.8 & 63.7 & 73.4 & 58.4 \\
H & 80 & German & Moderate & HC and IC & cat. 3 & 49.9 & 45.1 & 55.6 & 39.7 \\
\hline I & 80 & German & Moderate & IC & cat. 1 & 54.9 & 53.6 & 56.3 & 44.1 \\
J & 80 & German & Moderate & IC & cat. 2 & 58.0 & 56.7 & 59.2 & 46.9 \\
K & 80 & German & Moderate & IC & cat. 4 & 54.3 & 53.0 & 55.7 & 43.6 \\
\hline
\end{tabular}

Note: Refer to the notes in Table 11.

Table 12: Predictions of the overall care duration for females along representative characteristics.

\subsection{Practical implications}

Our work brings solid additional knowledge for designing LTC insurance solutions in Switzerland. First, by developing three distinct models, we take into account different perspectives. Our first model sheds light on the relevant characteristics when developing solutions that cover costs during the whole care duration irrespective of the type of care. The second and the third models give insights for more tailor-made products solely focusing on at-home or institutional care (Costa-Font and Courbage, 2012). Such solutions are often provided in specific private health or life insurance products (see, e.g., the Swiss company Helsana, 2019). This distinction is important as, for example, we find that the type of household significantly shapes the at-home care duration while its effect on the overall and the institutional care duration is not significant. Second, based on a reliable and comprehensive dataset, our predictions give a practical illustration of the changes in LTC durations along various socio-demographic characteristics. They provide strong insights for determining the cover period of LTC insurance products. In all predictions, we observe a mean time spent in dependence that is higher than the median. The presence of very high durations should not be neglected in policy discussions, governance and insurance coverage. The high volatility observed is critical for the pricing of insurance coverage. In fact, in the past, negative performance in and repricing of LTC products has been observed (e.g., Carrns, 2015).

Given the significantly higher costs yielded by institutional care and the limited availability of adequate infrastructure and professional caregivers, finding the optimal combination of both at-home and institutional care is an important concern for governments and policymakers. In fact, costs significantly differ between the two types of care (Buchmueller et al., 2005), adequate infrastructure is limited and the demand for professional caregivers is highly increasing. Initiatives are widely discussed by policymakers in many countries, like, e.g., the U.S. (Frank, 2012), France (Vasselle, 2008) and Germany (Reinhard, 2018). The results discussed in Section 4.2 imply co-existence of both substitution and non-substitution effects between the at-home and institutional care durations. Based our findings, the promotion of at-home care by local policies 
is an interesting strategy to contain the costs since it reduces the time spent in an institution. Indeed, the replacement of institutional care by at-home care can reduce the costs, cope with space limitations in institutions and partly overcome the shortage of caregivers (Stevenson and Grabowski, 2010). However, such policies should not be detrimental to the development of institutional care solutions in particular for those elderly without relatives or with more severe dependence. Our findings could also be further compared with and integrate individual preferences, as, e.g., the fact that elderly often prefer at-home care over institutional care, to develop satisfactory and sustainable solutions.

Finally, our findings can be useful beyond the framework of the present study based on Switzerland. In fact, the predicted care durations are close to the cover period currently found in practice. Benefits periods of three, four and five years are commonly proposed in the U.S. and French markets (Doty et al., 2015). From an insurer's perspective, defining the appropriate amount of benefits is just as critical and deserves further analysis. By covering the dependent population of an entire country, our findings are representative for the many heterogeneous paths in dependence. Further, insurance practitioners can apply our findings to more specific insurance portfolios when accepting selected individual profiles (cf. our predictions along individual characteristics). Furthermore, the econometric approach in the present study complements the semi-Markov approach taken by Fuino and Wagner (2018a). Both together lay the basis for insurance product design, pricing and underwriting factors in Switzerland and beyond. The thoughts laid out for insurers similarly apply to governments when reforming social LTC policies. Our findings on the care duration and the interactions between types of care together with the projected increasing elderly population (Fuino and Wagner, 2018b) can help to plan LTC resources.

\section{Conclusion}

In this paper, we empirically study the time spent in LTC on the basis of a comprehensive social insurance dataset comprising about 230000 recorded dependent persons in Switzerland. We specifically analyze the effect of socio-demographic factors on the care duration, the interaction between care provided at-home and in an institution as well as the evolution of the age at entry and the care duration. At the end of the work, we predict the mean time spent in dependence for selected profiles and discuss outcomes for insurers and policymakers. Using common survival analysis methods, we find that the age at entry, the gender, the linguistic region of residence, the acuity state at entry, the type of household, the types of care received as well as the pre-retirement income are significant for determining the LTC duration. At higher ages and at higher acuity levels, elderly spend less time in dependence what is in line with the increased mortality (Fuino and Wagner, 2018a). Further, we find that women stay on average about one year longer in dependence than men which follows conclusions from other studies (e.g., Mathers et al., 2001; Monod-Zorzi et al., 2007). We also observe a strong effect of the household composition on the at-home care duration. Living in a two-person household importantly reduces the duration in dependence. In fact, by receiving help from their partner, elderly register for dependence at higher ages and therefore spent less time therein (Freedman and Martin, 1998).

Since costs and infrastructure highly depend on the type of care that is needed, an important concern for LTC financing and planning stems from the interaction between at-home and institutional care. We find that receiving at-home care prior to institutional care can reduce 
by up to six months the overall care duration. However, after having received at-home care for one year or longer, any further increase in the at-home care duration will not reduce the institutional care duration. These outcomes show that, while the two types of care are substitutes for elderly having never received care at home, they become complements thereafter spawning the non-reducibility of institutional care at some stage. In fact, there are many factors impeding complete substitution of institutional care by at-home care, e.g., severely dependent elderly that require permanent supervision, highly qualified medical staff or specialized infrastructure (Callahan et al., 2012). This is even truer for elderly diagnosed with diseases requiring very particular care.

When evaluating the changes in the age at entry and the period spent in dependence over the past years, our results indicate that the age at entry has shifted towards higher ages along with the reported longevity gains for the Swiss population. We also reveal that the median time spent in dependence has not changed over the years with, e.g., an 80 years old man staying on average three years in dependence. Nonetheless, we remark that gender differences in the LTC duration remain significant along the whole period of observation. In the context of LTC, we therefore refute both theories of Fries (1980) and Olshansky et al. (1990) by stating that the time spent in dependence has not changed.

Various streams of research can extend the present study. First, our findings are limited to the set of available covariates and could be improved by considering further factors. For example, the pathology, informal care and the presence of children, exogeneous family characteristics and the cause of death have an effect on the elderly's path in dependence (Charles and Sevak, 2005; Marengoni et al., 2011; Fuino et al., 2019). Second, the interaction effect between at-home care and institutional care surely deserves further investigation in a separate study. Such analysis could also account for further types of care such as community and ambulatory care services (Feder et al., 2000). A deeper analysis of local policy usage could improve the understanding of the interaction between the LTC duration and cultural aspects. Finally, additional thoughts could build on the findings of the present work and Fuino and Wagner (2018a) in order to foster the development of LTC insurance products in Switzerland and beyond. In fact, while our results concentrate on the Swiss population, several aspects can be applied to further countries when taking into account the specific local LTC policies and insurance offers.

\section{Appendix}

\begin{tabular}{|c|c|c|c|c|c|c|}
\hline \multirow[b]{2}{*}{ Model } & \multicolumn{3}{|c|}{$\mathcal{D}_{1}$} & \multicolumn{3}{|c|}{$\mathcal{D}_{2}$} \\
\hline & $D$ & $D^{\mathrm{HC}}$ & $D^{\mathrm{IC}}$ & $D$ & $D^{\mathrm{HC}}$ & $D^{\mathrm{IC}}$ \\
\hline $\mathrm{AFT}$ & 1721602 & 100119 & 1685154 & 610895 & 66899 & 586426 \\
\hline Cox prop. hazard & 4149367 & 192674 & 4066822 & 1307692 & 123162 & 1257039 \\
\hline
\end{tabular}

Table 13: AIC values for the AFT and Cox proportional hazard rate approaches for the models presented in Tables 7 and 8. 


\begin{tabular}{lcccrr}
\hline \multicolumn{1}{c}{$D^{\mathrm{HC}}$} & $m_{D^{\mathrm{IC}}}$ & $m_{D^{\mathrm{IC}}, \text { lower }}$ & $m_{D^{\mathrm{IC}}, \text { upper }}$ & $\chi^{2}$ & $p$-value \\
\hline 0 month (baseline) & 32 & 32 & 32 & n.a. & n.a. \\
1 - 6 months & 31 & 29 & 33 & 3.136 & 0.077 \\
$7-12$ months & 29 & 26 & 32 & 20.905 & 0.000 \\
13 - 18 months & 30 & 27 & 33 & 16.223 & 0.000 \\
$19-24$ months & 33 & 28 & n.a. & 2.827 & 0.093 \\
\hline
\end{tabular}

Note: The columns " $m_{D^{\mathrm{IC}} \text {,lower" }}$ and " $m_{D^{\mathrm{IC}} \text {,upper" }}$ denote the lower respectively upper bounds of the $95 \%$ confidence intervals on the predicted median duration $m_{D^{\text {IC }}}$. Durations are expressed in months. The column " $\chi 2$ " reports the $\chi^{2}$-value of the log-rank test. "n.a." stands for not applicable.

Table 14: Numerical values for Figure 3 and log-rank tests between the Kaplan-Meier estimates of the survival curves for the institutional care duration for given at-home care durations $D^{\mathrm{HC}}$ and the baseline $\left(D^{\mathrm{HC}}=0\right)$.

\section{References}

Aalen, O. O., Ø. Borgan, and H. K. Gjessing, 2008, Survival and Event History Analysis. Statistics for Biology and Health. Springer, New York.

Becker, U. and H.-J. Reinhard, 2018, Long-Term Care in Europe: A Juridical Approach. Springer, Munich.

Biessy, G., 2016, A Semi-Markov Model with Pathologies for Long-Term Care Insurance, Working Paper, Université d'Évry Val d'Essonne.

Brown, J. R. and A. Finkelstein, 2007, Why is the Market for Long-Term Care Insurance so Small?, Journal of Public Economics, 91(10):1967-1991.

Brown, J. R. and A. Finkelstein, 2008, The Interaction of Public and Private Insurance : Medicaid and the Long-Term Care Insurance Market, American Economic Review, 98(3):10831102 .

Brown, J. R. and A. Finkelstein, 2009, The Private Market for Long-Term Care Insurance in the United States: A Review of the Evidence, Journal of Risk and Insurance, 76(1):5-29.

Buchmueller, T. C., T. Gilmer, and K. Harris, 2005, Long-Term Care Over an Uncertain Future: What Can Current Retirees Expect?, Inquiry, 42(4):335-350.

Cadena, M. and M. Denuit, 2016, Semi-Parametric Accelerated Hazard Relational Models with Applications to Mortality Projections, Insurance: Mathematics and Economics, 68:1-16.

Callahan, C. M., G. Arling, W. Tu, M. B. Rosenman, S. R. Counsell, T. E. Stump, and H. C. Hendrie, 2012, Transitions in Care for Older Adults with and without Dementia, Journal of the American Geriatrics Society, 60(5):813-820.

Carrns, A., 2015, Managing the Costs of Long-Term Care Insurance, New York Times, 2 Sept.

Chammartin, F., N. Probst-Hensch, J. Utzinger, and P. Vounatsou, 2016, Mortality Atlas of the Main Causes of Death in Switzerland, Swiss Medical Weekly, 146:1-13.

Charles, K. K. and P. Sevak, 2005, Can Family Caregiving Substitute for Nursing Home Care?, Journal of Health Economics, 24(6):1174-1190.

Choi, J. K. and E. Joung, 2016, The Association Between the Utilization of Long-Term Care Services and Mortality in Elderly Koreans, Archives of Gerontology and Geriatrics, 65(July):122127.

Collett, D., 2015, Modelling Survival Data in Medical Research. Chapman and Hall/CRC, London.

Colombo, F., A. Llena-Nozal, J. Mercier, and F. Tjadens, 2011, Help Wanted?: Providing and 
Paying for Long-Term Care. OECD Health Policy Studies, OECD Publishing.

Costa-Font, J. and C. Courbage, 2012, Financing Long-Term Care in Europe. Palgrave Macmillan, New York.

Courbage, C., G. Montoliu-Montes, and J. Wagner, 2018, Informal Care, Long-Term Care Insurance and Intra-Family Moral Hazard: Empirical Evidence from Italy and Spain, Working Paper, University of Lausanne.

Czado, C. and F. Rudolph, 2002, Application of Survival Analysis Methods to Long-Term Care Insurance, Insurance: Mathematics and Economics, 31(3):395-413.

D'Amico, G., M. Guillen, and R. Manca, 2009, Full Backward Non-Homogeneous Semi-Markov Processes for Disability Insurance Models: A Catalunya Real Data Application, Insurance: Mathematics and Economics, 45(2):173-179.

Doty, P., P. Nadash, and N. Racco, 2015, Long-Term Care Financing: Lessons From France, The Milbank Quarterly, 93(2):359-391.

Dutoit, L., S. Füglister-Dousse, and S. Pellegrini, 2016, Soins de Longue Durée dans les Cantons: un même Défi, Différentes Solutions. Observatoire Suisse de la Santé, Neuchâtel.

Eckert, J. K., L. A. Morgan, and N. Swamy, 2004, Preferences for Receipt of Care Among Community-Dwelling Adults, Journal of Aging \& Social Policy, 16(2):49-65.

Eugster, B., R. Lalive, A. Steinhauer, and J. Zweimüller, 2011, The Demand for Social Insurance: Does Culture Matter?, The Economic Journal, 121(556):413-448.

Feder, J., H. L. Komisar, and M. Niefeld, 2000, Long-Term Care In The United States: An Overview, Health Affairs, 19(3):40-56.

Federal Office of Public Health (FOPH), 2013, Mise en Oeuvre du Financement des Soins.

Fong, J. H., M. Sherris, and J. Yap, 2017, Forecasting Disability: Application of a Frailty Model, Scandinavian Actuarial Journal, 2017(2):125-147.

Frank, R. G., 2012, Long-Term Care Financing in the United States: Sources and Institutions, Applied Economic Perspectives and Policy, 34(2):333-345.

Freedman, V. A. and L. G. Martin, 1998, Understanding Trends in Functional Limitations Among Older Americans, American Journal of Public Health, 88(10):1457-1462.

Fried, L. P., C. M. Tangen, J. Walston, A. B. Newman, C. Hirsch, J. Gottdiener, T. Seeman, R. Tracy, W. J. Kop, G. Burke, and M. A. McBurnie, 2001, Frailty in Older Adults: Evidence for a Phenotype, The Journals of Gerontology Series A: Biological Sciences and Medical Sciences, 56(3):146-157.

Fries, J. F., 1980, Aging, Natural Death, and the Compression of Morbidity, The New England Journal of Medicine, 303(3):130-135.

Fries, J. F., 1989, The Compression of Morbidity: Near or Far?, The Milbank Quarterly, 67(3):208-232.

Fries, J. F., 2005, The Compression of Morbidity, The Milbank Quarterly, 83(4):801-823.

Fuino, M., I. Rudnytskyi, and J. Wagner, 2019, On the Socio-demographic and Medical Drivers of Long-Term Care Needs in Europe, Working Paper, University of Lausanne.

Fuino, M. and J. Wagner, 2018a, Long-Term Care Models and Dependence Probability Tables by Acuity Level: New Empirical Evidence from Switzerland, Insurance: Mathematics and Economics, 81:51-70.

Fuino, M. and J. Wagner, 2018b, Old-Age Care Prevalence in Switzerland: Drivers and Future Development, European Actuarial Journal, 8(2):321-362.

Fujisawa, R. and F. Colombo, 2009, The Long-Term Care Workforce: Overview and Strategies to Adapt Supply to a Growing Demand. OECD Publishing. 
Gentili, E., G. Masiero, and F. Mazzonna, 2017, The Role of Culture in Long-Term Care Arrangement Decisions, Journal of Economic Behavior \& Organization, 143:186-200.

Germain, C. M., E. Vasquez, J. A. Batsis, and D. R. McQuoid, 2016, Sex, Race and Age Differences in Muscle Strength and Limitations in Community Dwelling Older Adults: Data from the Health and Retirement Survey (HRS), Archives of Gerontology and Geriatrics, 65:98-103.

Gruenberg, E. M., 2005, The Failures of Success, The Milbank Quarterly, 83(4):779-800.

Harrington, D. P. and T. R. Fleming, 1982, A Class of Rank Test Procedures for Censored Survival Data, Biometrika, 69(3):553-566.

Helmchen, L. A. and A. T. Lo Sasso, 2015, The Causal Effects of Home Care Use on Institutional Long-Term Care Utilization and Expenditures, Health Economics, 24(1):4-17.

Helsana, 2019, Assurance des Soins de Longue Durée.

Hougaard, P., 1994, Heterogeneity Models of Disease Susceptibility, with Application to Diabetic Nephropathy, Biometrics, 50(4):1178-1188.

Jackson, C., 2016, flexsurv: A Platform for Parametric Survival Modeling in R, Journal of Statistical Software, 70(8):1-33.

Juma, S., M.-m. Taabazuing, and M. Montero-Odasso, 2016, Clinical Frailty Scale in an Acute Medicine Unit: a Simple Tool That Predicts Length of Stay, Canadian Geriatrics Journal, 19(2):34-39.

Junhua, W., C. Haozhe, and Q. Shi, 2013, Estimating Freeway Incident Duration using Accelerated Failure Time Modeling, Safety Science, 54:43-50.

Kaplan, E. L. and P. Meier, 1958, Nonparametric Estimation from Incomplete Observations, Journal of the American Statistical Association, 53(282):457-481.

Karadeniz, P. G. and I. Ercan, 2017, Examining Tests for Comparing Survival Curves with Right Censored Data, Statistics in Transition new series, 18(2):311-328.

Kaye, H. S., M. P. LaPlante, and C. Harrington, 2009, Do Noninstitutional Long-Term Care Services Reduce Medicaid Spending?, Health Affairs, 28(1):262-272.

Keiding, N., P. K. Andersen, and J. P. Klein, 1997, The Role of Frailty Models and Accelerated Failure Time Models in Describing Heterogeneity Due to Omitted Covariates, Statistics in Medicine, 16(2):215-224.

Klein, J. P. and M. L. Moeschberger, 1997, Survival Analysis Techniques for Censored and Truncated Data. Statistics for Biology and Health. Springer, New York.

Kramer, M., 1980, The Rising Pandemic of Mental Disorders and Associated Chronic Diseases and Disabilities, Acta Psychiatrica Scandinavica, 62(285):382-397.

Lafortune, G. and G. Balestat, 2007, Trends in Severe Disability Among Elderly People. OECD Publishing, Paris.

Lambert, P., D. Collett, A. Kimber, and R. Johnson, 2004, Parametric Accelerated Failure Time Models with Random Effects and an Application to Kidney Transplant Survival, Statistics in Medicine, 23(20):3177-3192.

Lee, K. H., V. Rondeau, and S. Haneuse, 2017, Accelerated Failure Time Models for SemiCompeting Risks Data in the Presence of Complex Censoring, Biometrics, 73(4):1401-1412.

Lin, D., 1998, Accelerated Failure Time Models for Counting Processes, Biometrika, 85(3):605618.

Llena-Nozal, A., J. Mercier, F. Tjadens, and F. Colombo, 2011, Help Wanted? OECD Publishing, Paris.

Lumley, T., P. Diehr, S. Emerson, and L. Chen, 2002, The Importance of the Normality As- 
sumption in Large Public Health Data Sets, Annual Reviews of Public Health, 23(1):151-169.

Marengoni, A., S. Angleman, R. Melis, F. Mangialasche, A. Karp, A. Garmen, B. Meinow, and L. Fratiglioni, 2011, Aging with Multimorbidity: A Systematic Review of the Literature, Ageing Research Reviews, 10(4):430-439.

Mathers, C., 1996, Trends in Health Expectancies in Australia 1981-1993, Journal of the Australian Population Association, 13(1):1-15.

Mathers, C., T. Vos, and C. Stevenson, 2001, The Burden of Disease and Injury in Australia, World Health Organisation, 23(1):1076-1084.

Mcauley, W. J. and R. Blieszner, 1985, Selection of Long-Term Care Arrangements by Older Community Residents, The Gerontologist, 25(2):188-193.

Mcgarry, K., 2011, Multiple Dimensions of Private Information: Evidence from the Long-Term Care Insurance Market, American Economic Review, 96(4):938-958.

Milliman, 2017, LTCI Survey Product Exhibit. Broker World, New York.

Mobley, L. R., E. Root, L. Anselin, N. Lozano-Gracia, and J. Koschinsky, 2006, Spatial Analysis of Elderly Access to Primary Care Services, International Journal of Health Geographics, $5(19): 1-17$.

Mommaerts, C., 2015, Long-Term Care Insurance and the Family, Working Paper, University of Wisconsin.

Monod-Zorzi, S., L. Seematter-Bagnoud, C. Büla, S. Pellegrini, and H. Jaccard Ruedin, 2007, Maladies Chroniques et Dépendance Fonctionnelle des Personnes Agées. Observatoire Suisse de la Santé, Neuchâtel.

Nichols, B. L., C. R. Davis, and D. R. Richardson, 2010, Global Nursing Issues. Springer, New York.

Nordman, E. C., 2016, The State of Long-Term Care Insurance: The Market, Challenges and Future Innovations. The Center for Insurance Policy and Research, New York.

OECD, 2017, Health at a Glance 201\%: OECD Indicators. OECD Publishing, Paris.

Olshansky, S., B. A. Carnes, and C. Cassel, 1990, In Search of Methuselah: Estimating the Upper Limits to Human Longevity, Science, 250(4981):634-640.

Orbe, J., E. Ferreira, and V. Núñez-Antón, 2002, Comparing Proportional Hazards and Accelerated Failure Time Models for Survival Analysis, Statistics in Medicine, 21(22):3493-3510.

Patel, K., R. Kay, and L. Rowell, 2006, Comparing Proportional Hazards and Accelerated Failure Time Models: an Application in Influenza, Pharmaceutical Statistics, 5(3):213-224.

Payne, G., A. Laporte, R. Deber, and P. C. Coyte, 2007, Counting Backward to Health Care's Future: Using Time-to-Death Modeling to Identify Changes in End-of-Life Morbidity and the Impact of Aging on Health Care Expenditures, The Milbank Quarterly, 85(2):213-257.

Pellegrini, S., J. R. Hélène, and C. Jeanrenaud, 2006, Coûts des Etablissements Médico-Sociaux et des Services d'Aide et de Soins à Domicile. Observatoire Suisse de la Santé, Neuchâtel.

Pepe, M. S. and T. R. Fleming, 1989, Weighted Kaplan-Meier Statistics : A Class of Distance Tests for Censored Survival Data, Biometrics, 45(2):497-507.

Pinquart, M. and S. Sörensen, 2011, Spouses, Adult Children, and Children-in-Law as Caregivers of Older Adults: A Meta-Analytic Comparison, Psychology and Aging, 26(1):1-14.

Planchet, F., Q. Guibert, and M. Schwarzinger, 2018, Mesure de l'Espérance de Vie en Dépendance Totale en France, Bulletin Français d'Actuariat, 18(35):133-159.

Rashwan, W., M. Ragab, W. Abo-Hamad, and A. Arisha, 2013, Evaluating Policy Interventions for Delay Discharge : A System Dynamics Approach, Proceedings of the 2013 Winter Simulation Conference, pages 2463-2474. 
Reinhard, H.-J., 2018, Long-Term Care in Germany, In U. Becker and H.-J. Reinhard, editors, Long-Term Care in Europe: A Juridical Approach, chapter 5. Springer, Munich.

Rodrigues, R., S. Ilinca, and A. E. Schmidt, 2018, Income-Rich and Wealth-Poor? The Impact of Measures of Socio-Economic Status in the Analysis of the Distribution of Long-Term Care Use Among Older People, Health Economics, 27(3):637-646.

Sakamoto, Y., M. Ishiguro, and G. Kitagawa, 1986, Akaike Information Criterion Statistics. Springer, New York.

Schulz, E., 2010, The Long-Term Care System for the Elderly in Germany. European Network of Economic Policy Research Institutes, Bruxelles.

Schwarzkopf, L., P. Menn, R. Leidl, S. Wunder, H. Mehlig, P. Marx, E. Graessel, and R. Holle, 2012, Excess Costs of Dementia Disorders and the Role of Age and Gender - An Analysis of German Health and Long-Term Care Insurance Claims Data, BMC Health Services Research, $12: 1-12$.

Stevenson, D. G. and D. C. Grabowski, 2010, Sizing Up The Market For Assisted Living, Health Affairs, 29(1):35-43.

Swindell, W. R., 2008, Accelerated Failure Time Models Provide a Useful Statistical Framework for Aging Research, Experimental Gerontology, 44(3):190-200.

Swiss Federal Social Insurance Office, 2018, Circular on the Individual Account. No. 318.106.02.

Swiss Re, 2014, How Will We Care? Finding Sustainable Long-Term Care Solutions for an Ageing World, Sigma, No 5/2014.

Therneau, T. M. and P. M. Grambsch, 2000, Modeling Survival Data: Extending the Cox Model. Springer, Berlin.

Tseng, Y. K., F. Hsieh, and J. L. Wang, 2005, Joint Modelling of Accelerated Failure Time and Longitudinal Data, Biometrika, 92(3):587-603.

United Nations, 2015, World Population Ageing 2015. Departement of Economic and Social Affairs, Population Division, New York.

United Nations, 2016, Human Development Report 2016. United Nations Development Programme, New York.

Vasselle, A., 2008, Rapport d'Information. Sénat Français, Paris.

Venables, W. N. and B. D. Ripley, 2002, Modern Applied Statistics with S-Plus. Springer, New York.

Viña, J. and A. Lloret, 2010, Why Women Have More Alzheimer's Disease than Men: Gender and Mitochondrial Toxicity of Amyloid- $\beta$ Peptide, Journal of Alzheimer's Disease, 20:527533.

Wei, L. J., 1992, The Accelerated Failure Time Model: A Useful Alternative to the Cox Regression Model in Survival Analysis, Statistics in Medicine, 11:1871-1879.

World Health Organization, 2014, Migration of Health Workers. WHO Document Production Services, Geneva. 